Article

\title{
Multi-Component One-Pot Synthesis and Antimicrobial Activities of 3-Methyl-1,4-diphenyl-7-thioxo-4,6,8,9-tetrahydro- pyrazolo[5,4-b]pyrimidino[5,4-e]pyridine-5-one and Related Derivatives
}

\section{Talaat I. El-Emary * and Shawkat A. Abd El-Mohsen}

Department of Chemistry, Faculty of Science, Assiut University, Assiut 71516, Egypt

* Author to whom correspondence should be addressed; E-Mail: emarytalaat@yahoo.com.

Received: 24 October 2012; in revised form: 25 November 2012 / Accepted: 27 November 2012 / Published: 6 December 2012

\begin{abstract}
The synthesis of 3-methyl-1,4-diphenyl-7-thioxo-4,6,8,9-tetrahydropyrazolo[5,4- $b$ ] pyrimidino[5,4-e]pyridine-5-one (6) was achieved by two different one-pot multi-component synthesis (one-pot three-component and one-pot four component synthesis). Mono and dialkylation of 6 under different conditions gave compounds 7-11. The hydrazine 12 produced from reaction of 9 with $\mathrm{N}_{2} \mathrm{H}_{4}$ was subjected to reactions with some aromatic aldehydes, ethyl acetoacetate, acetyl acetone, ethyl cyanoacetate and triethyl orthoformate to give 13-17, respectively. Compound 12 upon reaction with $\mathrm{CS}_{2}$, nitrous acid, benzoin, chloroacetone and phenacyl bromide gave 18,20,21,22. Alkylation of 18 with ethyl iodide, ethyl chloroacetate and phenacyl bromide gave 19a-c. The antibacterial and antifungal activities of selected derivatives were evaluated.
\end{abstract}

Keywords: one-pot reactions; pyrazolo[5,4-b]pyrimidino[5,4-e]pyridines; synthesis; anti-microbial activity

\section{Introduction}

Multicomponent domino reactions (MDRs), particularly those performed in aqueous media, have become an increasingly useful tool for the synthesis of chemically and biologically important compounds because of their convergence, atom economy, and other suitable characteristics from the point of view of green chemistry [1-10]. Pyrazoles are excellent precursors for the synthesis of condensed polyfunctionally substituted heterocycles [11-16]. Pyrazolo-annulated heterocycles such as 
pyrazolopyridopyrimidines have attracted considerable interest because their derivatives display a wide range of pharmacological activities, e.g., as anticonvulsants [17], antiproliferative agents [18], anti-inflammatories and analgesic agents [19]. In addition these types of compounds are inhibitors of cyclic guanosine-3',5'-monophosphate phosphodiesterase (cGMP PDE), and are thereby agents against erectile dysfunction [20]. They also show miscellaneous biological properties such as virucidal, anticancer, fungicidal, bactericidal and vasodilatory activities [21]. For all the benefits mentioned above and as part of our program investigating syntheses using pyrazole and fused pyrazoles that have biological importance [22-33], we report herein the synthesis of pyrazolo[5,4-b]pyrimidino[5,4-e]pyridinethiones in a one-pot four component environmental friendly method in light of recently reported methods [34-37].

\section{Results and Discussion}

\subsection{Chemistry}

We first describe a comparison between two methods for the construction of pyrazolo[5,4b]pyrimidino[5,4-e]pyridin-5-one (6), a one-pot four-component domino reaction of phenyl hydrazine (1), 3-aminocrotononitrile (2), benzaldehyde (3) and thiobarbituric acid (4) in water in the presence of one equivalent of $p$-toluenesulfonic acid ( $p$-TSA) (Method A) and the three-component reaction method involving 5-amino-3-methyl-1-phenylpyrazole (5), benzaldehyde (3) and thiobarbituric acid (4) in presence of $p$-TSA under solvent-free conditions [35] (Method B).

The one-pot four-component method A showed some crucial advantages, such as short reaction time, excellent yield and high purity, which makes it more efficient and broadly applicable. The percentage yield and the reaction time of the one-pot four-components method in comparison with the one-pot three-component one to produce compound 6 was found to be $91 / 66 \%$ and $1 \mathrm{~h} / 10 \mathrm{~h}$, respectively (Scheme 1).

Scheme 1. Synthetic pathways for compound 6.

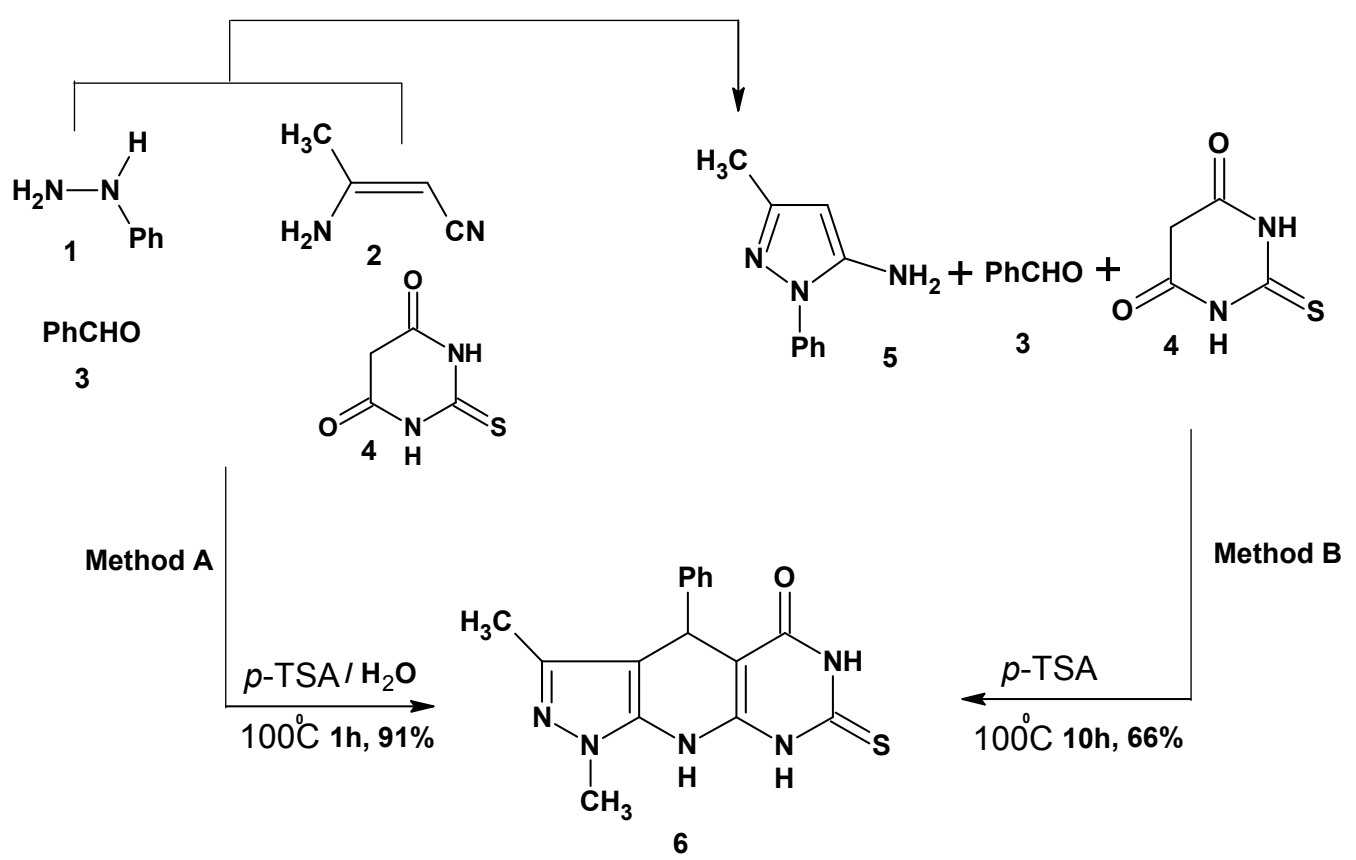


The plausible mechanism for the formation of compound $\mathbf{6}$ is proposed (Scheme 2) and it is in agreement with that proposed in the literature [38]. The domino sequence of reactions is presumably triggered by the formation of 5-amino-3-methyl-1-phenylpyrazole (5) from the acid-catalyzed reaction of phenylhydrazine (1) with 3-aminocrotononitrile (2). The readily formed 5 reacts in situ with benzaldehyde to afford intermediate $\mathbf{A}$. The later reacted with thiobarbituric acid under the acidic conditions to presumably furnish the intermediate $\mathbf{B}$, which subsequently undergoes annulations leading to the final intermediate $\mathbf{C}$. This final intermediate gave compound $\mathbf{6}$ upon losing a molecule of water.

Scheme 2. Mechanistic pathways for the formation of compound 6.
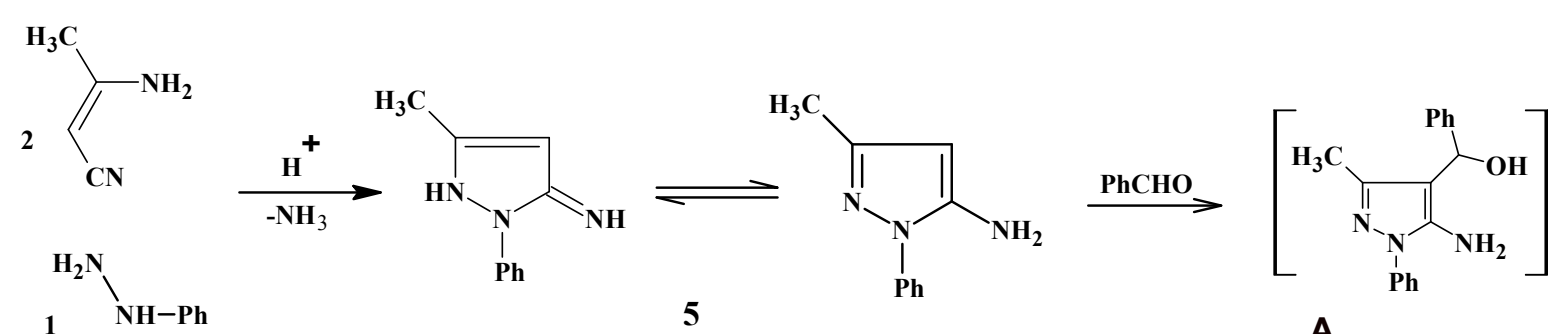

A
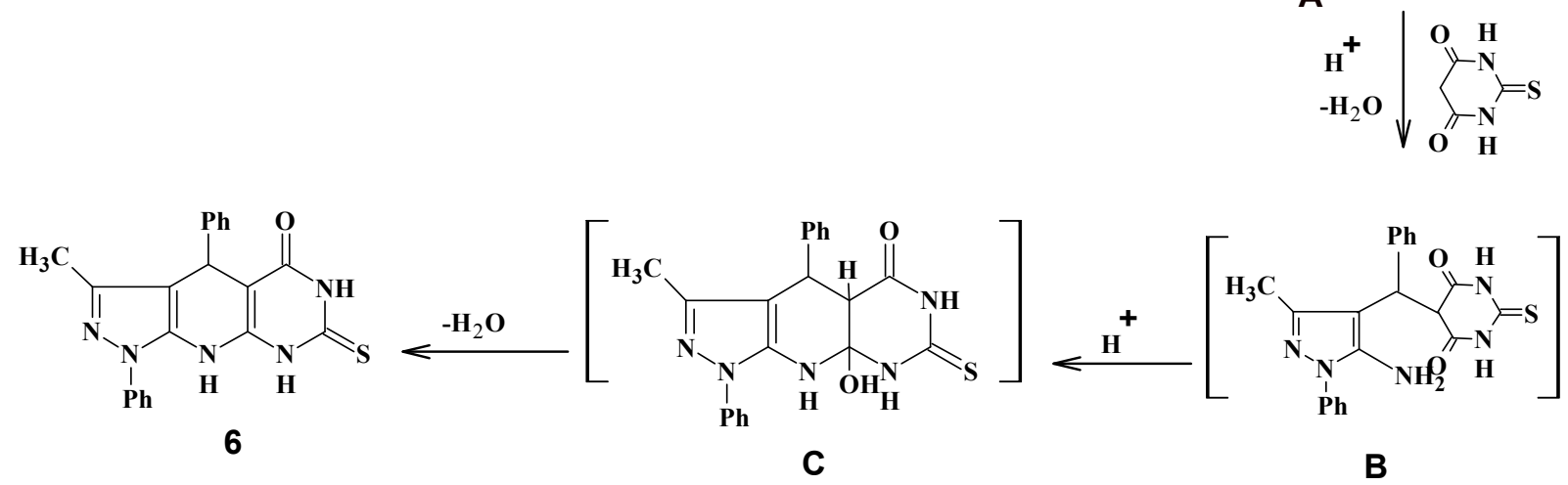

The structural features of compound 6 were elucidated from its spectral and analytical data. Thus, the IR spectrum revealed absorption bands at 3310, 3290, 3240, 1690, $1345 \mathrm{~cm}^{-1}$ characteristic for three $\mathrm{NH}, \mathrm{C}=\mathrm{O}$ and $\mathrm{C}=\mathrm{S}$ groups, respectively. The ${ }^{1} \mathrm{H}-\mathrm{NMR}$ (DMSO- $d_{6}$ ) displayed three $\mathrm{NH}$ singlets (exchangeable with $\mathrm{D}_{2} \mathrm{O}$ ) at $13.8,12.2$ and $9.4 \mathrm{ppm}$. A characteristic singlet peak for the pyridine CH-4 appeared at $5.57 \mathrm{ppm}$. Several alkylated derivatives were obtained from the versatile compound 6. Thus, upon treatment of $\mathbf{6}$ with ethyl iodide, ethyl chloroacetate, phenacyl bromide, chloroacetone, chloroacetamide and chloroacetonitrile in the presence of anhydrous sodium acetate in refluxing ethanol, the S-alkylated derivatives $7 \mathbf{a}-\mathbf{f}$ were obtained. The IR spectral data of compounds $7 \mathbf{a}-\mathbf{f}$ displayed no absorption band for $\mathrm{C}=\mathrm{S}$ and showed bands at $1280-1345 \mathrm{~cm}^{-1}$ confirming the S-alkylation, as well as, compounds $7 \mathbf{b}-\mathbf{e}$ revealed absorption bands for $\mathrm{C}=\mathrm{O}$ at $1690-1735 \mathrm{~cm}^{-1}$. A characteristic absorption band at $2240 \mathrm{~cm}^{-1}$ has been observed for $\mathrm{CN}$, confirming the structure of $7 \mathbf{f}$. Regioselective dialkylation reactions of compound $\mathbf{6}$ using two equivalent of ethyl iodide indicate that, in the introduction of the second ethyl group into the pyrimidine ring there is a competition between $\mathrm{N} 1$ or $\mathrm{N} 3$ and revealed that it depends on the base conditions used in the reactions. Thus, refluxing in ethanolic $\mathrm{NaOH}$ for $4 \mathrm{~h}$ (Method A) afforded the two isomeric compounds 8 and 9 in $63 \%$ and 25\% yield, respectively, whereas treatment of 6 with two equivalents of ethyl iodide in DMF in the presence of anhydrous $\mathrm{K}_{2} \mathrm{CO}_{3}$ at room temperature (Method $\mathrm{B}$ ) afforded in high yield the isomer 9 and only traces of the isomer 8 (Scheme 2). The reaction was monitored by TLC. The reaction mixture was 
chromatographically work up over silica gel using Pet. ether (b.p. $\left.60-80{ }^{\circ} \mathrm{C}\right)$ and ethyl acetate $(1: 1)$ as eluent, to afforded two products in pure form. A crystalline solid, m.p. $162-164{ }^{\circ} \mathrm{C}$ obtained in the first fraction was characterized as 6-ethyl-7-ethylthio-3-methyl-1,4-diphenyl-4,6,9-trihydropyrazolo[5,4$b]$ pyrimidino[5,4-e] pyridine-5-one (9). The second fraction afforded a solid, m.p. 190-192 ${ }^{\circ} \mathrm{C}$, which was identified as 8-ethyl-7-ethylthio-3-methyl-1,4-diphenyl-4,6,9-trihydropyrazolo[5,4- $b$ ] pyrimidino[5,4-e]pyridin-5-one (8). By TLC comparison, data analysis and melting point determination, it was found that one of the products 9 was identical to that obtained from Method B. The chemical shift for the pyrimidinone carbonyl is markedly affected by the nature of the adjacent nitrogen [39-43]. The ${ }^{13} \mathrm{C}$-NMR spectral data of compound 9 showed that the $\delta$ values of the pyrimidine $\mathrm{C}=\mathrm{O}$ at $165.82 \mathrm{ppm}$ suggest that $\mathrm{N}-3$ near to $\mathrm{C}=\mathrm{O}$ is $\mathrm{sp}^{3}$-hybridized (pyrrole type) and different from the $\mathrm{C}=\mathrm{O}$ adjacent to a $\mathrm{sp}^{2}$-hybridized nitrogen (pyridine type) in compound 8, which appears at $178.14 \mathrm{ppm}[42,43]$. Moreover, reaction of $\mathbf{6}$ with highly electron withdrawing aromatic halo compounds such as 2,4-dinitrochlorobenzene in DMF at room temperature yielded the S-aryl derivative 10, whereas, upon heating, benzothiazolo derivative $\mathbf{1 1}$ was obtained via ring closure reaction with the N3-pyrimidine ring [44]. As chemical evidence, the formation of compound 11 was achieved through heating of a sample of $\mathbf{1 0}$ in DMF. The structures of $\mathbf{1 0}$ and $\mathbf{1 1}$ were deduced from their satisfactory spectral and analytical data, for example, the mass spectrum of compound $\mathbf{1 1}$ showed its correct parent ion peak at $m / z 506\left(\mathrm{M}^{+}, 100 \%\right)$ (Scheme 3).

Scheme 3. Synthetic pathways for compounds 7a-f, 8, 9, 10 and 11.

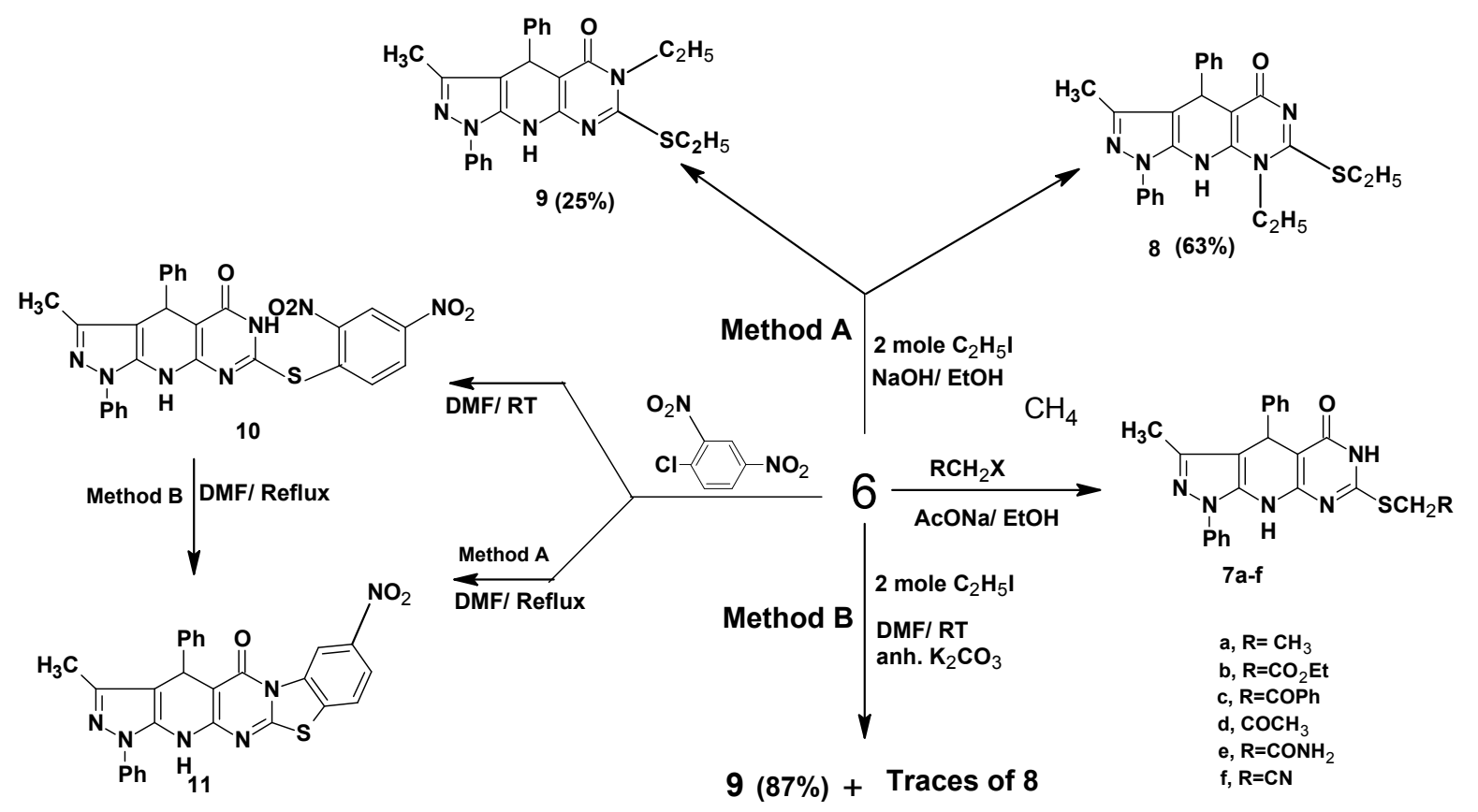

The sulfur-free compound 12, which was identified as 6-ethyl-7-hydrazino-3-methyl-1,4-diphenyl4,6,9-trihydropyrazolo[5,4- $b]$ pyrimidino[5,4-e]pyridin-5-one, was obtained in excellent yield (85\%) via nucleophilic displacement of the thioethyl group in compound 9 with hydrazine in boiling ethanol (Scheme 4). 
Scheme 4. Synthetic pathways for compounds 12-17.

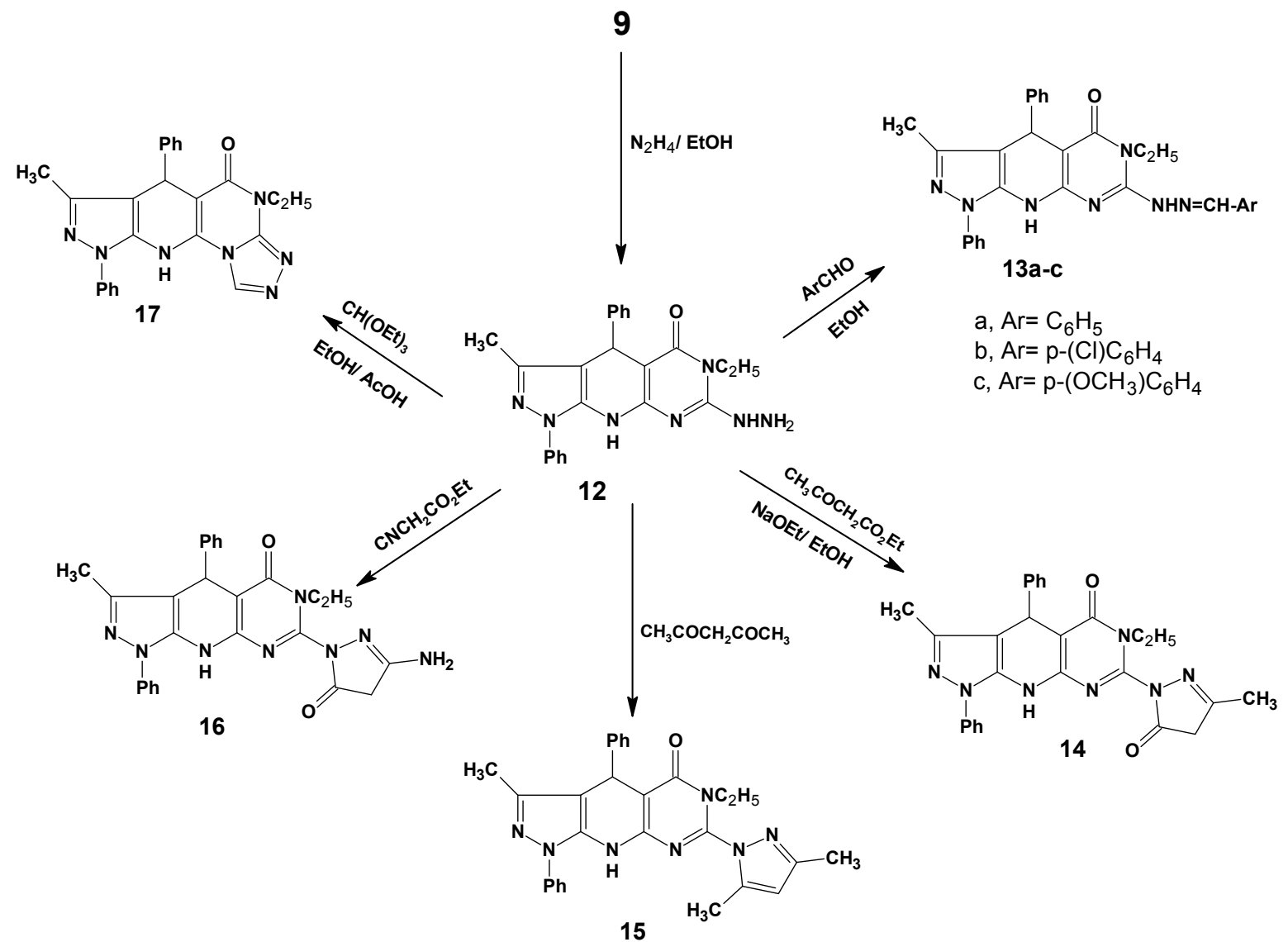

The hydrazine derivative $\mathbf{1 2}$ was used as the key intermediate for the synthesis of some polyheterocyclic compounds. Thus, heating of compound 12 with some aromatic aldehydes namely, benzaldehyde, 4-chlorobenzaldeyde and 4-methoxybenzaldeyde in the presence of a few drops of acetic acid in ethanol resulted in the formation of the corresponding hydrazones 13a-c. The structure of compounds 13a-c was characterized by the disappearance of the $\mathrm{NHNH}_{2}$ group and revealed in each case two bands at 3425-3250 and 3170-3100 $\mathrm{cm}^{-1}$ assignable to two NH groups. Also, their ${ }^{1} \mathrm{H}-\mathrm{NMR}$ spectra showed the presence of the azomethine and two NH protons at 8.9-9.5 and 10.85-12.75 ppm, respectively. On the other hand, upon heating the hydrazino compound 12 with ethyl acetoacetate, acetylacetone and ethyl cyanoacetate in ethanolic sodium ethoxide solution, the $N$-pyrazolo derivatives 14-16 were produced. For example, the ${ }^{1} \mathrm{H}-\mathrm{NMR}$ spectrum for compound 15 revealed a singlet at $6.15 \mathrm{ppm}$ due to the $4-H$-pyrazole moiety. The ${ }^{13} \mathrm{C}$-NMR spectral data displayed two characteristic singlets at 151.12 and $152.5 \mathrm{ppm}$ for $\mathrm{C} 3$ and $\mathrm{C} 5$ of the pyrazole nucleus, respectively, which was in agreement with the literature value [45]. The formation of the tetracyclic pyrazolo[5,4-b]1,2,4-triazolo[4',3'-2,1]pyrimidino[5,6-e]pyridine (17) was achieved upon reaction of 12 with triethyl orthoformate in ethanol in presence of a few drops of acetic acid (Scheme 4). The ${ }^{1} \mathrm{H}-\mathrm{NMR}$ spectrum of compound $\mathbf{1 7}$ revealed the disappearance of $\mathrm{NHNH}_{2}$ signals and appearance of a singlet signal at $8.55 \mathrm{ppm}$ due to the triazole $\mathrm{CH}$.

Furthermore, the interaction of the hydrazine 12 with $\mathrm{CS}_{2}$ in pyridine furnished the angular tetracyclic pyrazolo[5,4-b]1,2,4-triazolo[4',3'-2,1]pyrimidino[5,6-e]pyridin-3(2H)-thione (18) in good yield (Scheme 5). 
Scheme 5. Synthetic pathways for compounds 18-22a,b.

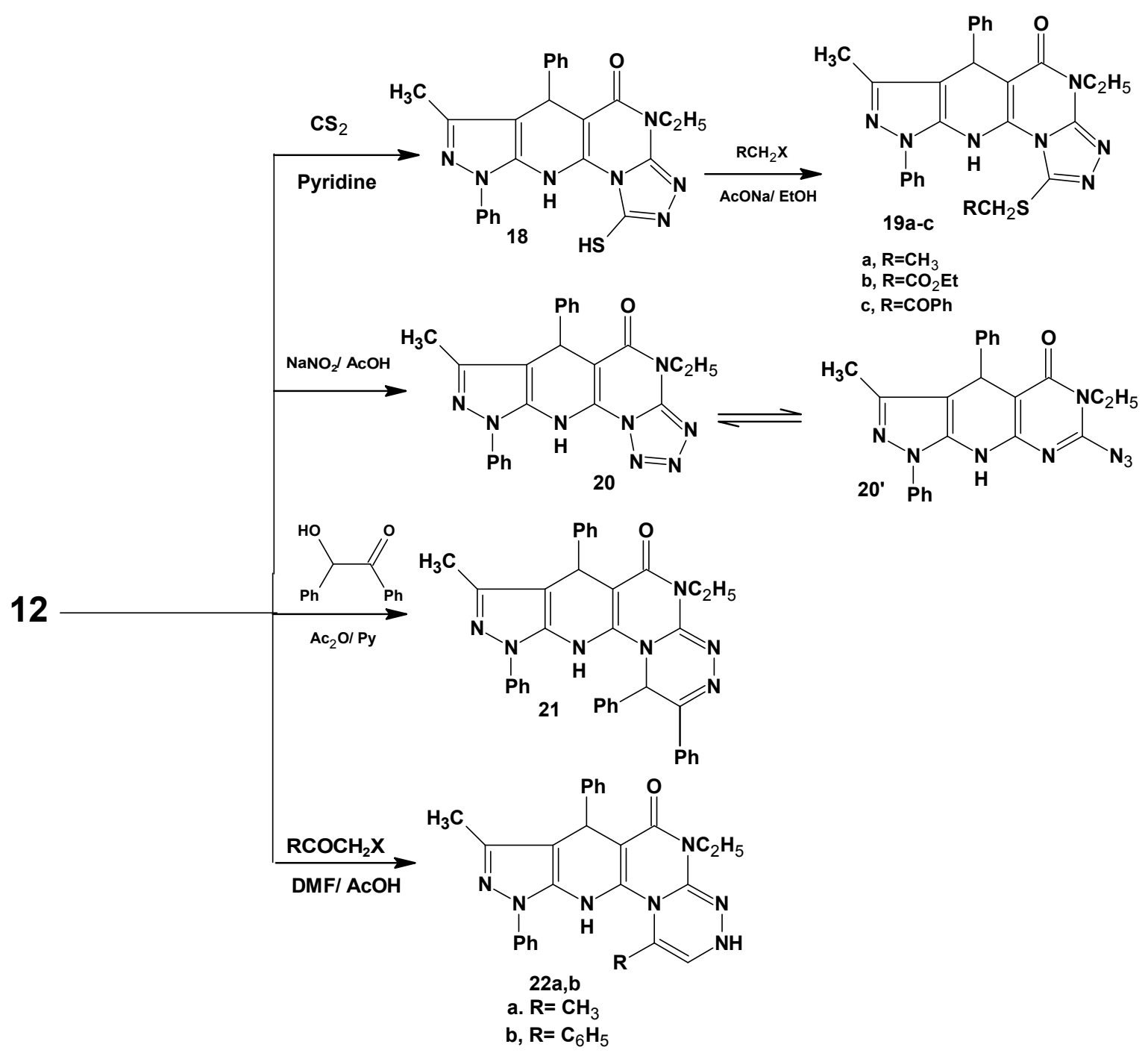

The thione 18 was easily converted into a series of S-alkylated derivatives 19a-c upon treatment with ethyl iodide, ethyl chloroacetate and phenacyl bromide in the presence of anhydrous sodium acetate in refluxing ethanol, respectively. As well, the reaction of $\mathbf{1 1}$ with sodium nitrite in acetic acid at $0{ }^{\circ} \mathrm{C}$, led to the formation of the pyrazolotetrazolopyrimidinopyridine $\mathbf{2 0}$ in $\mathbf{7 5 \%}$ yield. This new ring system is in equilibrium with the corresponding 2-azido tautomer $\mathbf{2 0}^{\prime}$ [46], which was confirmed by a characteristic absorption peak of the azido group at $2250 \mathrm{~cm}^{-1}$ in the IR. The tetracyclic pyrazolotriazinopyrimidinopyridine compounds $\mathbf{2 1}$ and 22a,b were obtained in good to high yield when compound 12 was allowed to react with benzoin in acetic anhydride- pyridine mixture and/or with some $\alpha$-haloketones (chloroacetone and phenacyl bromide) in $\mathrm{DMF} / \mathrm{AcOH}$, respectively (Scheme 5). The structures of compounds 21 and 22a,b were confirmed by spectral data. For example, the ${ }^{1} \mathrm{H}-\mathrm{NMR}$ spectrum of compound 22b revealed a singlet at 9.3 due to $\mathrm{CH}$-triazine and displayed two broad singlets $\left(\mathrm{D}_{2} \mathrm{O}\right.$-exchangable) at 10.75 and 11.25 due to $\mathrm{NH}$ groups. The mass spectra of compound 22b showed its correct parent ion peak at $m / z 513\left(\mathrm{M}^{+}, 100 \%\right)$. 


\subsection{Biological Results}

Using the agar well-diffusion method [47], ten selected derivatives (compounds 6, 7a, 9, 11, 12, 13b, 17, 19c, 20, and 22a) were evaluated for their antibacterial and antifungal activities. Thus, these compounds were screened against Staphylococcus aureus, Bacillus cereus, Micrococcus luteus as a Gram positive bacteria and Escherichia coil, Pseudomonas aeruginosa and Serratia marcescens as Gram negative bacteria using chloramphenicol as control (Table 1). The MIC results indicated that three of the tested compounds $(\mathbf{1 1}, 19$ and 22a) showed significant activity against Staphylococcus aureus (Table 1). Compounds 12 and 19c showed highly significant activity against Bacillus cereus and moderate activity against Micrococcus luteus (Table 1). Compound 22a revealed moderate activity against Staphylococcus aureus and Pseudomonas aeruginosa (Table 1). The rest of tested compounds were inactive against all bacterial strains used.

Table 1. Antibacerial activity data [inhibition zone in $\mathrm{mm} / \mathrm{MICs}$ (in $\mathrm{mM}$ ).

\begin{tabular}{ccccccc}
\hline & \multicolumn{7}{c}{ Diameter of the inihibition zone (mm) } \\
Compound & \multicolumn{7}{c}{ MIC (mM) } \\
\cline { 2 - 7 } No. & S. aureus & S. cereus & M. luteus & E. coli & p. aeruginosa & S. marcescens \\
& AUMC & AUMC & AUMC & AUMC & AUMC & AUMC \\
& $B .54$ & $B .52$ & $B .112$ & $B .53$ & $B .73$ & $B .55$ \\
\hline 6 & - & - & - & - & - & - \\
$7 \mathrm{a}$ & - & - & - & - & - & - \\
9 & - & - & - & - & - & - \\
11 & $8(2.5)$ & - & - & - & - & - \\
12 & - & $10(1.25)$ & $18(20)$ & - & - & - \\
$13 b$ & - & - & - & - & - & - \\
17 & - & - & - & - & - & - \\
$19 \mathrm{c}$ & $\mathbf{8}(1.25)$ & $10(0.15)$ & $10(5)$ & - & - & - \\
20 & - & - & - & - & - & - \\
$22 \mathrm{a}$ & $8(5)$ & - & - & - & $11(20)$ & - \\
CHL & $10(0.08)$ & $12(1.25)$ & $12(2.5)$ & $10(0.08)$ & $12(0.3)$ & $13(1.25)$ \\
\hline
\end{tabular}

$\mathrm{CHL}=$ chloramphenicol as control.

The same compounds $(6,7 \mathbf{a}, 9,11,12,13 \mathbf{b}, 17,19 \mathbf{c}, 20$ and 22a) were screened for their antifungal activities against six fungal strains: (Candida albicans AUMC No. 418, Trichophyton rubrum AUMC No. 1804, Aspergillus flavus AUMC No. 1276, Fusarium oxysporum AUMC No. 5119, Scopulariopsis brevicaulis AUMC No. 729, Geotrichum candidum AUMC No. 226) using clotrimazole as control. The results are listed in Table 2. The MIC values showed that compounds 22a, 12 and $\mathbf{1 7}$ exhibit moderate to low activity against Candida albicans AUMC No. 418. Compounds 19c, 12 and 11 showed moderate to low activity against Geotrichum candidum AUMC No. 226. Compounds 17, 19c, 12 and 10 revealed moderate to low activity against Trichophyton rubrum AUMC No. 1804. Compounds 19c, 12 and 17 showed moderate to poor activity against Aspergillus flavus AUMC No. 1276. Compounds 19c, 12 and 17 showed moderate to poor activity against Fusarium oxysporum AUMC No. 5119. Compounds 19c, 12, 17 and 11 showed moderate to poor activity against Scopulariopsis brevicaulis AUMC No. 729. The rest of tested compounds were inactive against all fungal strains used. 
Table 2. Antifungal activity data [inhibition zone in $\mathrm{mm} / \mathrm{MICs}$ (in $\mathrm{mM}$ ).

\begin{tabular}{ccccccc}
\hline & \multicolumn{7}{c}{ Diameter of the inihibition zone (mm) } \\
Compound & \multicolumn{7}{c}{ MIC (mM) } \\
\cline { 2 - 7 } No. & C. albicans & G.candidum & T. rubrum & A.flavus & F. oxysporum & S. brevicaylis \\
& AUMC & AUMC & AUMC & AUMC & AUMC & AUMC \\
& 418 & 226 & 1804 & 3214 & 5119 & 729 \\
\hline 6 & - & - & - & - & - & - \\
$7 \mathrm{a}$ & - & - & - & - & - & - \\
9 & - & - & - & - & - & - \\
11 & - & $11(20)$ & $8(20)$ & - & - & $10(20)$ \\
12 & $9(2.5)$ & $15(5)$ & $10(10)$ & $10(2.5)$ & $16(20)$ & $20(5)$ \\
$13 b$ & - & - & - & - & - & - \\
17 & $10(20)$ & - & $24 p . i(20)$ & $10(20)$ & $8(20)$ & $12(10)$ \\
$19 c$ & $9(2.5)$ & $16(2.5)$ & $12(5)$ & $14(2.5)$ & $10(10)$ & $28(5)$ \\
20 & - & - & - & - & - & - \\
$22 a$ & $10(0.6)$ & - & - & - & - & - \\
CLO & $12(0.08)$ & $14(0.08)$ & $35(0.08)$ & $15(0.15)$ & $14(0.15)$ & 24 p.i (0.3) \\
\hline
\end{tabular}

$\mathrm{CLO}=$ Clotrimazole as control; $\mathrm{p} . \mathrm{i}=$ Partial inhibition.

\section{Experimental}

\subsection{General}

All melting points were determined on a Kofler melting point apparatus. IR spectra were recorded on a Pye Unicam SP3-100 spectrophotometer using the $\mathrm{KBr}$ wafer technique. The ${ }^{1} \mathrm{H}-\mathrm{NMR}$ spectra were recorded on a Bruker ARX 200 spectrometer $\left(200 \mathrm{MHz}\right.$ for ${ }^{1} \mathrm{H}$ and $50 \mathrm{MHz}$ for $\left.{ }^{13} \mathrm{C}\right)$ at the Faculty of Science, University of King Saoud, Saudi Arabia, Riyadh and on a Jeol LA $400 \mathrm{MHz}$ (400 MHz for ${ }^{1} \mathrm{H}, 100 \mathrm{MHz}$ for the ${ }^{13} \mathrm{C}$ ) at Assiut University, ${ }^{1} \mathrm{H}$ and ${ }^{13} \mathrm{C}$ NMR chemical shifts $(\delta$ ) were reported in parts per million (ppm) and were referenced to the solvent peak; $\mathrm{CDCl}_{3}$ ( $7.26 \mathrm{ppm}$ for ${ }^{1} \mathrm{H}$ and $76.90 \mathrm{ppm}$ for ${ }^{13} \mathrm{C}$ ) and DMSO- $\mathrm{d}_{6}\left(2.50 \mathrm{ppm}\right.$ for ${ }^{1} \mathrm{H}$ and $39.70 \mathrm{ppm}$ for $\left.{ }^{13} \mathrm{C}\right)$. Multiplicities are represented by s (singlet), d (doublet), $\mathrm{t}$ (triplet), q (quartet) and m (multiplet). Coupling constants $(J)$ are reported in Hertz $(\mathrm{Hz})$. Mass spectra were taken on a JEOL JMS600 spectrometer at an ionizing potential of $70 \mathrm{eV}$ (EI) at Assiut University. Elemental analyses were carried out using a Perkin-Elmer $240 \mathrm{C}$ Micro analyzer at Assiut University and they were found to be within $\pm 0.4 \%$ of the theoretical values.

3.2. Synthesis of 3-Methyl-1,4-diphenyl-7-thioxo-4,6,8,9-tetrahydropyrazolo[5,4-b]pyrimidino[5,4e]pyridin-5-one (6)

Method A: A mixture of thiobarbituric acid (1.44 g, $0.01 \mathrm{~mol})$, 3-methyl-1-phenyl-1H-pyrazol-5amine $(1.73 \mathrm{~g}, 0.01 \mathrm{~mol})$, benzaldehyde $(1.1 \mathrm{~g}, 0.012 \mathrm{~mol})$ and $p$-TSA $(1 \mathrm{~g}, 0.05 \mathrm{~mol})$ was heated at $100{ }^{\circ} \mathrm{C}$ for $10 \mathrm{~h}$ (monitored by TLC). After cooling, the reaction mixture was washed with water $(20 \mathrm{~mL})$ and residue recrystallized from $\mathrm{EtOH}$ to afford the pure product $\mathbf{6}$ as a yellow powder. Yield: 66\%. Mp 202-204 ${ }^{\circ} \mathrm{C}$. IR (KBr) cm ${ }^{-1}: 3240,3290,3310(3 \mathrm{NH}), 1690(\mathrm{C}=\mathrm{O}), 1620(\mathrm{C}=\mathrm{N}), 1345(\mathrm{C}=\mathrm{S})$. ${ }^{1} \mathrm{H}-\mathrm{NMR}\left(\mathrm{DMSO}_{\mathrm{d}}\right)$ ) 2.75 (s, 3H, $\mathrm{CH}_{3}$ ), 5.57 (s, 1H, CH pyridine), 7.25-7.98 (m, 10H, Ar-H), 9.45 
(s, 1H, NH), 12.20 (br s, 1H, NH), 13.80 (br s, 1H, NH). ${ }^{13} \mathrm{C}-\mathrm{NMR}\left(\mathrm{DMSO}-\mathrm{d}_{6}\right) \delta(\mathrm{ppm}): 11.93\left(\mathrm{CH}_{3}\right)$, $33.8\left(\mathrm{CH} \mathrm{sp}^{3}\right), 102.11-160.12\left(17 \mathrm{C}, \mathrm{sp}^{2}\right.$ carbon atoms), $161.73(\mathrm{C}=\mathrm{O}), 174.45(\mathrm{C}=\mathrm{S})$. Anal. Calcd. For $\mathrm{C}_{21} \mathrm{H}_{17} \mathrm{~N}_{5} \mathrm{OS}$ (387.45): C, 65.10; H, 4.42; N, 18.08; S, 8.28. Found: C, 65.15; H, 4.38; N, 18.04; S, 8.23.

Method B: A mixture of phenylhydrazine (1 mmol), 3-aminocrotononitrile $(1 \mathrm{mmol})$ and $p$-TSA $(0.5 \mathrm{mmol})$ in water $(10 \mathrm{~mL})$ was added to benzaldehyde $(1 \mathrm{mmol})$ and thiobarbituric acid $(1 \mathrm{mmol})$ and the reaction mixture was heated under reflux at $100{ }^{\circ} \mathrm{C}$ for $1 \mathrm{~h}$, after completion of the reaction (TLC), the reaction mixture cooled to room temperature, the precipitate filtered off and washed with water and recrystallized from EtOH to afford the pure product 6 as a yellow powder. Yield: 91\%. All of spectral and physical data were in agreement with that described in method A.

\subsection{General Procedure for the Preparation of $7 \mathbf{a}-\mathbf{f}$}

A mixture of 6 (3.8 g, $0.01 \mathrm{~mol})$, ethyl iodide and/or $\alpha$-haloketone compound $(0.01 \mathrm{~mol})$ in ethanol $(50 \mathrm{~mL})$ was refluxed in the presence of anhydrous sodium acetate $(0.9 \mathrm{~g}, 0.011 \mathrm{~mol})$ for $4 \mathrm{~h}$. The solid product separated from the hot mixture was filtered off, washed with water and recrystallized from the proper solvent.

7-Ethylthio-3-methyl-1,4-diphenyl-4,6,9-trihydropyrazolo[5,4-b]pyrimidino[5,4-e]pyridin-5-one (7a). Yellow crystals. Yield: $72 \%$. Mp. $135-137^{\circ} \mathrm{C}$ (acetic acid). IR (KBr) cm ${ }^{-1}: 3220,3315(2 \mathrm{NH}), 1690$ $(\mathrm{C}=\mathrm{O}), 1620(\mathrm{C}=\mathrm{N}) .{ }^{1} \mathrm{H}-\mathrm{NMR}\left(\mathrm{DMSO}_{6}\right) \delta(\mathrm{ppm}): 1.55\left(\mathrm{t}, J=7.4 \mathrm{~Hz}, 3 \mathrm{H}, \mathrm{CH}_{3}\right), 2.78\left(\mathrm{~s}, 3 \mathrm{H}, \mathrm{CH}_{3}\right)$, 3.65 (q, $\left.J=7.4 \mathrm{~Hz}, 2 \mathrm{H}, \mathrm{CH}_{2}\right), 5.55(\mathrm{~s}, 1 \mathrm{H}, \mathrm{CH}), 7.2-7.8(\mathrm{~m}, 10 \mathrm{H}, \mathrm{Ar}-\mathrm{H}), 8.9(\mathrm{~s}, 1 \mathrm{H}, \mathrm{NH}), 10.2(\mathrm{~s}, 1 \mathrm{H}$, $\mathrm{NH}) .{ }^{13} \mathrm{C}-\mathrm{NMR}\left(\mathrm{DMSO}_{6}\right) \delta(\mathrm{ppm}): 12.03\left(\mathrm{CH}_{3}\right), 15.34\left(\mathrm{CH}_{3}\right), 24.12\left(\mathrm{CH}_{2}\right), 33.5\left(\mathrm{CH} \mathrm{sp}^{3}\right)$, 103.15-160.12 (18C, $\mathrm{sp}^{2}$ carbon atoms), 161.73( $\mathrm{C}=\mathrm{O}$ ), Anal. Calcd. for $\mathrm{C}_{23} \mathrm{H}_{21} \mathrm{~N}_{5} \mathrm{OS}$ (415.52): 66.48; H, 5.09; N, 16.85; S, 7.71. Found: C, 66.61; H, 5.13; N, 16.79; S, 7.68.

Ethyl-2-(3-methyl-5-oxo-1,4-diphenyl-4,6,9-trihydro-pyrazolo[5,4-b]pyrimidino[5,4-e]pyridin-7ylthio)acetate (7b). Yellow crystals. Yield: $67 \%$. Mp. $95-97{ }^{\circ} \mathrm{C}$ (methanol). IR (KBr) $\mathrm{cm}^{-1}: 3290$, $3310(2 \mathrm{NH}), 1735(\mathrm{C}=\mathrm{O}), 1690(\mathrm{C}=\mathrm{O}), 1620(\mathrm{C}=\mathrm{N}) .{ }^{1} \mathrm{H}-\mathrm{NMR}\left(\mathrm{DMSO}_{\mathrm{d}}\right) \delta(\mathrm{pp}): \mathrm{m} 1.27(\mathrm{t}, J=7.4 \mathrm{~Hz}$, $\left.3 \mathrm{H}, \mathrm{CH}_{3}\right), 2.80\left(\mathrm{~s}, 3 \mathrm{H}, \mathrm{CH}_{3}\right), 3.95\left(\mathrm{~s}, 2 \mathrm{H}, \mathrm{SCH}_{2}\right), 4.25\left(\mathrm{q}, J=7.4 \mathrm{~Hz}, 2 \mathrm{H}, \mathrm{CH}_{2}\right), 5.52(\mathrm{~s}, 1 \mathrm{H}, \mathrm{CH})$, 7.2-7.9 (m, 10H, Ar-H), $8.7(\mathrm{~s}, 1 \mathrm{H}, \mathrm{NH}), 11.1(\mathrm{~s}, 1 \mathrm{H}, \mathrm{NH}) .{ }^{13} \mathrm{C}-\mathrm{NMR}\left(\mathrm{CDCl}_{3}\right) \delta(\mathrm{ppm}): 12.03\left(\mathrm{CH}_{3}\right)$, $15.34\left(\mathrm{CH}_{3}\right), 29.12\left(\mathrm{CH}_{2}\right), 33.55\left(\mathrm{CH} \mathrm{sp}{ }^{3}\right), 61.11\left(\mathrm{CH}_{2}\right), 103.15-160.12$ (18C, sp ${ }^{2}$ carbon atoms), $161.73(\mathrm{C}=\mathrm{O}), 170.25\left(\mathrm{C}=\mathrm{O}\right.$ ester). Anal. Calcd. for: $\mathrm{C}_{25} \mathrm{H}_{23} \mathrm{~N}_{5} \mathrm{O}_{3} \mathrm{~S}$ (473.54): $\mathrm{C}, 63.41 ; \mathrm{H}, 4.90 ; \mathrm{N}$, 14.79; S, 6.77. Found: C, 63.49; H, 5.01; N, 14.82; S, 6.86.

3-Methyl-7-(2-oxo-2-phenylethylthio)-1,4-diphenyl-4,6,9-trihydropyrazolo [5,4-b]pyrimidino[5,4-e]pyridin-5-one (7c). Light yellow crystals. Yield: $71 \%$. Mp. $170-172{ }^{\circ} \mathrm{C}$ (methanol). IR $(\mathrm{KBr}) \mathrm{cm}^{-1}$ : 3245, $3290(2 \mathrm{NH}), 1,685(\mathrm{C}=\mathrm{O}), 1690(\mathrm{C}=\mathrm{O}), 1620(\mathrm{C}=\mathrm{N})$; ${ }^{1} \mathrm{H}-\mathrm{NMR}\left(\mathrm{DMSO}-\mathrm{d}_{6}\right) \delta(\mathrm{ppm}): 2.72(\mathrm{~s}$, $\left.3 \mathrm{H}, \mathrm{CH}_{3}\right), 4.19$ (s, 2H, SCH $\left.2 \mathrm{CO}\right), 5.57$ (s, 1H, CH), 6.9-8.6 (m, 15H, Ar-H), 8.84 (s, 1H, NH), 10.73 $(\mathrm{s}, 1 \mathrm{H}, \mathrm{NH}) ;{ }^{13} \mathrm{C}-\mathrm{NMR}\left(\mathrm{DMSO}-\mathrm{d}_{6}\right) \delta(\mathrm{ppm}): 12.93\left(\mathrm{CH}_{3}\right), 34.42\left(\mathrm{CH} \mathrm{sp}{ }^{3}\right), 35.60\left(\mathrm{CH}_{2}\right), 102.75-160.30$ (18C, sp ${ }^{2}$ carbon atoms), $162.03(\mathrm{C}=\mathrm{O}), 195.30(\mathrm{C}=\mathrm{O})$ Anal. Calcd. for: $\mathrm{C}_{29} \mathrm{H}_{23} \mathrm{~N}_{5} \mathrm{O}_{2} \mathrm{~S}$ (505.58): C, 68.89; H, 4.59; N, 13.85; S, 6.34. Found: C, 68.97; H, 4.64; N, 13.91; S, 6.29. 
3-Methyl-7-(2-oxopropylthio)-1,4-diphenyl-4,6,9-trihydropyrazolo[5,4-b]pyrimidino[5,4-e]pyridin-5-one (7d). Yellow crystals. Yield: 65\%. Mp. 223-225 ${ }^{\circ} \mathrm{C}$ (ethanol); IR (KBr) cm ${ }^{-1}: 3195,3290(2 \mathrm{NH})$, $1685(\mathrm{C}=\mathrm{O}), 1700(\mathrm{C}=\mathrm{O}) ;{ }^{1} \mathrm{H}-\mathrm{NMR}\left(\mathrm{CDCl}_{3}\right) \delta(\mathrm{ppm}): 2.75\left(\mathrm{~s}, 3 \mathrm{H}, \mathrm{CH}_{3}\right), 3.35\left(\mathrm{~s}, 3 \mathrm{H}, \mathrm{COCH}_{3}\right), 4.10$ $\left(\mathrm{s}, 2 \mathrm{H}, \mathrm{SCH}_{2}\right), 5.60(\mathrm{~s}, 1 \mathrm{H}, \mathrm{CH}), 7.35-7.95(\mathrm{~m}, 10 \mathrm{H}, \mathrm{Ar}-\mathrm{H}), 9.15(\mathrm{~s}, 1 \mathrm{H}, \mathrm{NH}), 10.2(\mathrm{~s}, 1 \mathrm{H}, \mathrm{NH})$. ${ }^{13} \mathrm{C}-\mathrm{NMR}\left(\mathrm{CDCl}_{3}\right) \delta(\mathrm{ppm}): 11.93\left(\mathrm{CH}_{3}\right), 29.32\left(\mathrm{CH}_{3}\right), 33.82(\mathrm{CH} \mathrm{sp}), 38.22\left(\mathrm{CH}_{2}\right), 103.15-160.12$ (18C, sp ${ }^{2}$ carbon atoms), $161.53(\mathrm{C}=\mathrm{O}), 202.11(\mathrm{C}=\mathrm{O})$. Anal. Calcd. for: $\mathrm{C}_{24} \mathrm{H}_{21} \mathrm{~N}_{5} \mathrm{O}_{2} \mathrm{~S}$ (443.52): $\mathrm{C}$, 64.99; H, 4.77; N, 15.79; S, 7.23. Found: C, 65.13; H, 4.81; N, 15.83; S, 7.19.

2-(3-Methyl-5-oxo-1,4-diphenyl-4,6,9-trihydropyrazolo[5,4-b]pyrimidino[5,4-e]pyridin-7-ylthio)acetamide (7e). Orange crystals. Yield: $69 \%$. Mp. $166-168{ }^{\circ} \mathrm{C}(\mathrm{DMF}) . \mathrm{IR}(\mathrm{KBr}) \mathrm{cm}^{-1}: 3420,3400\left(\mathrm{NH}_{2}\right), 3250$, $3310(2 \mathrm{NH}), 1685(\mathrm{C}=\mathrm{O}), 1665(\mathrm{C}=\mathrm{O}), 1690(\mathrm{C}=\mathrm{O}) .{ }^{1} \mathrm{H}-\mathrm{NMR}\left(\mathrm{CDCl}_{3}\right) \delta(\mathrm{ppm}): 2.65\left(\mathrm{~s}, 3 \mathrm{H}, \mathrm{CH}_{3}\right)$, 3.70 (s, 2H, $\mathrm{SCH}_{2}$ ), 5.30 (br.s, $\left.2 \mathrm{H}, \mathrm{NH}_{2}\right), 6.15$ (s, 1H, CH), 7.35-7.96 (m, 10H, Ar-H), $8.95(\mathrm{~s}, 1 \mathrm{H}$, $\left.\mathrm{NH}), 10.78(\mathrm{~s}, 1 \mathrm{H}, \mathrm{NH}) ;{ }^{13} \mathrm{C}-\mathrm{NMR}\left(\mathrm{CDCl}_{3}\right) \delta(\mathrm{ppm}): 12.11\left(\mathrm{CH}_{3}\right), 30.34\left(\mathrm{CH}_{2}\right), 34.52(\mathrm{CH} \mathrm{sp})^{3}\right)$, 104.85-159.90 (18C, sp ${ }^{2}$ carbon atoms), $162.30(\mathrm{C}=\mathrm{O}), 171.45(\mathrm{C}=\mathrm{O})$ Anal. Calcd. for $\mathrm{C}_{23} \mathrm{H}_{20} \mathrm{~N}_{6} \mathrm{O}_{2} \mathrm{~S}$ (444.50): C, 62.15; H, 4.54; N, 18.91; S, 7.21. Found: C, 62.29; H, 4.61; N, 19.03; S, 7.15.

2-(3-Methyl-5-oxo-1,4-diphenyl-4,6,9-trihydropyrazolo[5,4-b]pyrimidino[5,4-e]pyridin-7-ylthio)

ethanenitrile (7f). Orange crystals. Yield: $61 \%$. Mp. $147-149{ }^{\circ} \mathrm{C}$ (ethanol). IR (KBr) cm ${ }^{-1}: 3215,3200$ $\left.(2 \mathrm{NH}), 2,240(\mathrm{C}=\mathrm{N}), 1665(\mathrm{C}=\mathrm{O}) ;{ }^{1} \mathrm{H}-\mathrm{NMR}\left(\mathrm{DMSO}_{-} \mathrm{d}_{6}\right)\right) \delta(\mathrm{ppm}): 2.70\left(\mathrm{~s}, 3 \mathrm{H}, \mathrm{CH}_{3}\right), 3.90(\mathrm{~s}, 2 \mathrm{H}$, $\left.\mathrm{SCH}_{2} \mathrm{CO}\right), 6.12(\mathrm{~s}, 1 \mathrm{H}, \mathrm{CH}), 7.55-8.22(\mathrm{~m}, 10 \mathrm{H}, \mathrm{Ar}-\mathrm{H}), 9.20(\mathrm{~s}, 1 \mathrm{H}, \mathrm{NH}), 10.6(\mathrm{~s}, 1 \mathrm{H}, \mathrm{NH}) .{ }^{13} \mathrm{C}-\mathrm{NMR}$ $\left(\mathrm{DMSO}_{6}\right) \delta(\mathrm{ppm}): 12.31\left(\mathrm{CH}_{3}\right), 15.38\left(\mathrm{CH}_{2}\right), 33.84(\mathrm{CH} \mathrm{sp}), 104.80-159.95\left(18 \mathrm{C}, \mathrm{sp}^{2}\right.$ carbon atoms), $161.90(\mathrm{C}=\mathrm{O})$. Anal. Calcd. for $\mathrm{C}_{23} \mathrm{H}_{18} \mathrm{~N}_{6} \mathrm{OS}$ (426.49): C, 64.77; H, 4.25; N, 19.70; S, 7.52. Found: C, 64.89; H, 4.31; N, 19.61; S, 7.61.

3.4. 8-Ethyl-7-ethylthio-3-methyl-1,4-diphenyl-4,6,9-trihydropyrazolo[5,4-b]pyrimidino[5,4-e]pyridin5-one (8) and 6-Ethyl-7-ethylthio-3-methyl-1,4-diphenyl-4,6,9-trihydropyrazolo[5,4-b]pyrimidino[5,4e]pyridin-5-one (9)

Method A: A mixture of 6 (3.8 g, $0.01 \mathrm{~mol})$, ethyl iodide $(3.1 \mathrm{~g}, 0.021 \mathrm{~mol})$ and sodium hydroxide $(0.8 \mathrm{~g}, 0.02 \mathrm{~mol})$ in ethanol $(50 \mathrm{~mL})$ was refluxed for $4 \mathrm{~h}$. Excess ethanol was distilled off and the residue obtained on cooling was found to be mixture of two products as evidenced by the TLC. The residue was subjected to column chromatography over silica gel using mixtures of pet-ether/ethyl acetate of increasing polarity of eluent to yield compound $\mathbf{9}$ as an orange solid and compound $\mathbf{8}$ as a yellow solid in the second fraction.

8-Ethyl-7-ethylthio-3-methyl-1,4-diphenyl-4,6,9-trihydropyrazolo[5,4-b]pyrimidino[5,4-e]pyridin-5-one (8). Yield: 63\%. Mp. 190-192 ${ }^{\circ} \mathrm{C}$. IR (KBr) cm ${ }^{-1}: 3190(\mathrm{NH}), 1700(\mathrm{C}=\mathrm{O}),{ }^{1} \mathrm{H}-\mathrm{NMR}$ (DMSO-d $\left.{ }_{6}\right)$ $\delta$ (ppm): 1.00 (t, $\left.J=7.4 \mathrm{~Hz}, 3 \mathrm{H}, \mathrm{NCH}_{2} \underline{\mathrm{CH}}_{3}\right), 1.35$ (t, $\left.J=7.4 \mathrm{~Hz}, 3 \mathrm{H}, \mathrm{SCH}_{2} \underline{\mathrm{CH}}_{3}\right), 2.68\left(\mathrm{~s}, 3 \mathrm{H}, \mathrm{CH}_{3}\right)$, 3.15 (q, $\left.J=7.4 \mathrm{~Hz}, 2 \mathrm{H}, \mathrm{NCH}_{2} \mathrm{CH}_{3}\right), 3.95$ (q, $\left.J=7.4 \mathrm{~Hz}, 2 \mathrm{H}, \underline{\mathrm{SCH}}_{2} \mathrm{CH}_{3}\right), 5.65$ (s, 1H, CH), 7.40-7.80 (m, $10 \mathrm{H}, \mathrm{Ar}-\mathrm{H}), 10.45$ (s, $1 \mathrm{H}, \mathrm{NH}) .{ }^{13} \mathrm{C}-\mathrm{NMR}$ (DMSO-d $) \delta(\mathrm{ppm}): 12.50\left(\mathrm{CH}_{3}\right), 12.88\left(\mathrm{CH}_{3}\right), 15.09\left(\mathrm{CH}_{3}\right)$, $26.13\left(\mathrm{~S}-\mathrm{CH}_{2}\right), 37.04\left(\mathrm{CH}_{2}\right), 39.25\left(\mathrm{CH} \mathrm{sp}^{3}\right), 105.77-162.15\left(18 \mathrm{C}, \mathrm{sp}^{2}\right.$ carbon atoms $), 178.14(\mathrm{C}=\mathrm{O})$. 
6-Ethyl-7-ethylthio-3-methyl-1,4-diphenyl-4,6,9-trihydropyrazolo[5,4-b]pyrimidino[5,4-e]pyridin-5-one (9). Yield: 25\%. Mp. 162-164 ${ }^{\circ} \mathrm{C}$. IR (KBr) cm ${ }^{-1}: 3235(\mathrm{NH}), 1695(\mathrm{C}=\mathrm{O}),{ }^{1} \mathrm{H}-\mathrm{NMR}\left(\mathrm{DMSO}-\mathrm{d}_{6}\right)$ $\delta$ (ppm): 1.32 (t, $\left.J=7.4 \mathrm{~Hz}, 3 \mathrm{H}, \mathrm{NCH}_{2} \underline{\mathrm{CH}}_{3}\right), 1.65$ (t, $J=7.4 \mathrm{~Hz}, 3 \mathrm{H}, \mathrm{SCH}_{2} \underline{\mathrm{CH}_{3}}$ ), $2.78\left(\mathrm{~s}, 3 \mathrm{H}, \mathrm{CH}_{3}\right)$, 3.45 (q, $\left.J=7.4 \mathrm{~Hz}, 2 \mathrm{H}, \underline{\mathrm{NCH}}_{2} \mathrm{CH}_{3}\right), 4.05$ (q, $\left.J=7.4 \mathrm{~Hz}, 2 \mathrm{H}, \underline{\mathrm{SCH}}_{2} \mathrm{CH}_{3}\right), 5.75(\mathrm{~s}, 1 \mathrm{H}, \mathrm{CH}), 7.40-7.96$ $(\mathrm{m}, 10 \mathrm{H}, \mathrm{Ar}-\mathrm{H}), 10.26$ (s, 1H, NH). ${ }^{13} \mathrm{C}-\mathrm{NMR}\left(\mathrm{DMSO}-\mathrm{d}_{6}\right) \delta(\mathrm{ppm}): 11.81\left(\mathrm{CH}_{3}\right), 12.56\left(\mathrm{CH}_{3}\right), 14.88$ $\left(\mathrm{CH}_{3}\right), 24.28\left(\mathrm{~S}-\mathrm{CH}_{2}\right), 33.14\left(\mathrm{CH}_{2}\right), 35.22\left(\mathrm{CH} \mathrm{sp}{ }^{3}\right), 103.77-160.15$ (18C, sp ${ }^{2}$ carbon atoms), 163.16 $(\mathrm{C}=\mathrm{O})$; Anal. Calcd. for: $\mathrm{C}_{25} \mathrm{H}_{25} \mathrm{~N}_{5} \mathrm{OS}(443.56) \mathrm{C}, 67.69 ; \mathrm{H}, 5.68$; N, 15.79; S, 7.23. Found: C, 67.81; $\mathrm{H}, 5.61 ; \mathrm{N}, 15.81 ; \mathrm{S}, 7.16$.

Method B: A mixture of $6(1.90 \mathrm{~g}, 0.005 \mathrm{~mol})$ and ethyl iodide $(1.55 \mathrm{~g}, 0.01 \mathrm{~mol})$ in DMF $(30 \mathrm{~mL})$ in the presence of anhydrous potassium carbonate $(0.6 \mathrm{~g}, 0.002 \mathrm{~mol})$ was stirred at room temperature for $10 \mathrm{~h}$. The reaction mixture was cooled, poured onto ice cold water. The solid product separated was filtered off, washed with water and recrystallized from dioxane to yield compound 9. All of the physical and analytical data were in agreement with those of the product obtained using method A.

\subsection{7-(2,4-Dinitrophenylthio)-3-methyl-1,4-diphenyl-4,6,9-trihydropyrazolo[5,4-b]pyrimidino[5,4-e]} pyridin-5-one (10)

A mixture of 6 (1.14 g, $0.003 \mathrm{~mol})$ and 2,4-dinitrochlorobenzene (0.6 g, $0.003 \mathrm{~mol})$ in DMF (40 mL) was stirred at room temperature for $5 \mathrm{~h}$. The reaction mixture was cooled to $0{ }^{\circ} \mathrm{C}$ for $2 \mathrm{~h}$, a yellow fine crystals was obtained, it was filtered off dried and recrystallized from dioxane. Yield: $68 \%$. Mp. 251-253 ${ }^{\circ} \mathrm{C}$. IR (KBr) cm ${ }^{-1}:$ 3325, $3295(2 \mathrm{NH}), 1690$ (C=O), ${ }^{1} \mathrm{H}-\mathrm{NMR}$ (DMSO-d (D) $\delta(\mathrm{ppm}): 2.75$ (s, $\left.3 \mathrm{H}, \mathrm{CH}_{3}\right), 5.65(\mathrm{~s}, 1 \mathrm{H}, \mathrm{CH}), 7.14-8.92(\mathrm{~m}, 13 \mathrm{H}, \mathrm{Ar}-\mathrm{H}), 10.26(\mathrm{~s}, 1 \mathrm{H}, \mathrm{NH}), 11.35(\mathrm{~s}, 1 \mathrm{H}, \mathrm{NH})$. ${ }^{13} \mathrm{C}-\mathrm{NMR}\left(\mathrm{DMSO}-\mathrm{d}_{6}\right) \delta(\mathrm{ppm}): 11.90\left(\mathrm{CH}_{3}\right), 34.35\left(\mathrm{CH} \mathrm{sp}^{3}\right), 103.77-161.17$ (25C, $\mathrm{sp}^{2}$ carbon atoms), $162.99(\mathrm{C}=\mathrm{O})$; Anal. Calcd. for: $\mathrm{C}_{27} \mathrm{H}_{19} \mathrm{~N}_{7} \mathrm{O}_{5} \mathrm{~S}$ (553.54) C, 58.58; H, 3.46; N, 17.71; S, 5.79. Found: C, $58.96 ; \mathrm{H}, 3.69 ; \mathrm{N}, 17.97 ; \mathrm{S}, 6.14$.

\subsection{3-Methyl-8-nitro-1,4-diphenyl-4,6,13-trihydrobenzothiazolo[3',2'-2,1]pyrimidino[5,4-e] pyrazolo-} [5,4-b]pyridin-5-one (11)

Method A: A mixture of $6(0.38 \mathrm{~g}, 0.001 \mathrm{~mol})$ and 2,4-dinitrochlorobenzene $(0.2 \mathrm{~g}, 0.001 \mathrm{~mol})$ in DMF $(20 \mathrm{~mL})$ was refluxed for $2 \mathrm{~h}$. The reaction mixture was concentrated for its half volume and allowed to cool, yellowish crystals was obtained, filtered off, dried and recrystallized from acetic acid. Yield: 59\%. Mp. $>300{ }^{\circ} \mathrm{C}$. IR $(\mathrm{KBr}) \mathrm{cm}^{-1}: 3180(\mathrm{NH}), 1685(\mathrm{C}=\mathrm{O}),{ }^{1} \mathrm{H}-\mathrm{NMR}\left(\mathrm{DMSO}-\mathrm{d}_{6}\right) \delta(\mathrm{ppm})$ : $2.79\left(\mathrm{~s}, 3 \mathrm{H}, \mathrm{CH}_{3}\right), 4.95$ (s, 1H, CH), 7.10-7.85 (m, 13H, Ar-H), 9.95 (s, 1H, NH). ${ }^{13} \mathrm{C}-\mathrm{NMR}\left(\mathrm{DMSO}-\mathrm{d}_{6}\right)$ $\delta$ (ppm): $12.30\left(\mathrm{CH}_{3}\right), 34.15\left(\mathrm{CH} \mathrm{sp}^{3}\right), 102.57-162.11$ (25C, sp ${ }^{2}$ carbon atoms), $163.09(\mathrm{C}=\mathrm{O})$; Anal. Calcd. for: $\mathrm{C}_{27} \mathrm{H}_{18} \mathrm{~N}_{6} \mathrm{O}_{3} \mathrm{~S}$ (506.53) C, 64.02; H, 3.58; N, 16.59; S, 6.33. Found: C, 64.42; H, 3.99; N, $16.91 ; \mathrm{S}, 6.64$. MS $m / z(\%) 506.12\left(\mathrm{M}^{+}, 100\right)$.

Method B: A sample of $\mathbf{1 0}(0.5 \mathrm{~g}, 0.001 \mathrm{~mol})$ was heated under reflux in DMF (30 mL) for $2 \mathrm{~h}$. The separated product 11 was obtained and purified as described in method A. All physical and analytical data of the two final products obtained from both methods A and B are identical. 
3.7. 6-Ethyl-7-hydrazino-3-methyl-1,4-diphenyl-4,6,9-trihydropyrazolo[5,4-b]pyrimidino[5,4-e]pyridin-5-one (12)

A mixture of 9 (1.76 g, $0.004 \mathrm{~mol})$ and hydrazine hydrate $(15 \mathrm{~mL})$ in absolute ethanol $(40 \mathrm{~mL})$ was refluxed for $12 \mathrm{~h}$. The reaction mixture was poured onto ice. The product was isolated and crystallized from acetic acid as white needles. Yield: 85\%. Mp. 280-281 ${ }^{\circ} \mathrm{C}, \mathrm{IR}(\mathrm{KBr}) \mathrm{cm}^{-1}: 3440,3335,3210$ $\left(\mathrm{NH}, \mathrm{NH}_{2}\right), 1695(\mathrm{C}=\mathrm{O}) ;{ }^{1} \mathrm{H}-\mathrm{NMR}\left(\mathrm{DMSO}_{-} \mathrm{d}_{6}\right) \delta(\mathrm{ppm}): 1.23\left(\mathrm{t}, J=7.4 \mathrm{~Hz}, 3 \mathrm{H}, \mathrm{NCH}_{2} \underline{\mathrm{CH}}_{3}\right), 2.81$ (s, $\left.3 \mathrm{H}, \mathrm{CH}_{3}\right), 3.35$ (q, $\left.J=7.4 \mathrm{~Hz}, 2 \mathrm{H}, \mathrm{NCH}_{2} \mathrm{CH}_{3}\right), 4.95$ (s, 2H, $\mathrm{NH}_{2}, \mathrm{D}_{2} \mathrm{O}$ exchangeable), 6.04 (s, 1H, $\mathrm{CH}), 7.55-8.22(\mathrm{~m}, 10 \mathrm{H}, \mathrm{Ar}-\mathrm{H}), 9.82\left(\mathrm{~s}, 1 \mathrm{H}, \mathrm{NH}, \mathrm{D}_{2} \mathrm{O}\right.$ exchangeable), $10.55(\mathrm{~s}, 1 \mathrm{H}, \mathrm{NH}) .{ }^{13} \mathrm{C}-\mathrm{NMR}$ $\left(\mathrm{DMSO}_{6}\right) \delta(\mathrm{ppm}): 12.21\left(\mathrm{CH}_{3}\right), 13.01\left(\mathrm{CH}_{3}\right), 30.58\left(\mathrm{CH}_{2}\right), 34.92\left(\mathrm{CH} \mathrm{sp}{ }^{3}\right), 102.65-162.95\left(18 \mathrm{C}, \mathrm{sp}^{2}\right.$ carbon atoms), $163.56(\mathrm{C}=\mathrm{O})$. Anal. Calcd. for: $\mathrm{C}_{23} \mathrm{H}_{23} \mathrm{~N}_{7} \mathrm{O}$ (413.47): C, 66.81; H, 5.61; N, 23.71; Found: C, 66.95; H, 5.67; N, 23.79.

\subsection{General Procedure for the Preparation of 13a-c}

A mixture of compound $12(0.413 \mathrm{~g}, 0.001 \mathrm{~mol})$ and the appropriate aromatic aldehyde $(0.001 \mathrm{~mol})$ was stirred under reflux in ethanol $(30 \mathrm{ml})$ in the presence of a few drops of glacial acetic acid for $5 \mathrm{~h}$. The reaction mixture was allowed to cool to room temperature, poured into water, whereby a solid formed that was filtered off and crystallized from an appropriate solvent to produce 13a-c in good yields.

6-Ethyl-3-methyl-1,4-diphenyl-4,6,9-trihydropyrazolo[5,4-b]pyrimidino[5,4-e]pyridin-5-one-7benzaldehyde hydrazone (13a). Pale white crystals from acetic acid. Yield: 70\%. Mp. 268-269 ${ }^{\circ} \mathrm{C}$; IR $(\mathrm{KBr}) \mathrm{cm}^{-1}:$ 3425, $3295(2 \mathrm{NH}), 1675(\mathrm{C}=\mathrm{O}), 1625(\mathrm{C}=\mathrm{N}),{ }^{1} \mathrm{H}-\mathrm{NMR}\left(\mathrm{DMSO}-\mathrm{d}_{6}\right) \delta(\mathrm{ppm}): 1.20(\mathrm{t}$, $\left.J=7.4 \mathrm{~Hz}, 3 \mathrm{H}, \mathrm{NCH}_{2} \underline{\mathrm{CH}}_{3}\right), 2.78\left(\mathrm{~s}, 3 \mathrm{H}, \mathrm{CH}_{3}\right), 3.30$ (q, $\left.J=7.4 \mathrm{~Hz}, 2 \mathrm{H}, \underline{\mathrm{NCH}}_{2} \mathrm{CH}_{3}\right), 6.10(\mathrm{~s}, 1 \mathrm{H}, \mathrm{CH})$, 7.55-8.25 (m, 15H, Ar-H), 8.95 (s,1H, azomethine proton), 11.10 (brs, 1H, NH, D $2 \mathrm{O}$ exchangeable), 12.75 (brs, $1 \mathrm{H}, \mathrm{NH}, \mathrm{D}_{2} \mathrm{O}$ exchangeable); ${ }^{13} \mathrm{C}-\mathrm{NMR}\left(\mathrm{DMSO}_{6}\right) \delta(\mathrm{ppm}): 11.91\left(\mathrm{CH}_{3}\right), 12.99\left(\mathrm{CH}_{3}\right)$, $30.81\left(\mathrm{CH}_{2}\right), 35.20\left(\mathrm{CH} \mathrm{sp}^{3}\right), 102.65-162.95\left(21 \mathrm{C}, \mathrm{sp}^{2}\right.$ carbon atoms $), 162.66(\mathrm{C}=\mathrm{O}), 164.11(\mathrm{~N}=\mathrm{C})$. Anal. Calcd. for: $\mathrm{C}_{30} \mathrm{H}_{27} \mathrm{~N}_{7} \mathrm{O}$ (501.58): C, 71.84; H, 5.43; N, 19.55; Found: C, 71.93; H, 5.59; N, 19.61.

6-Ethyl-3-methyl-1,4-diphenyl-4,6,9-trihydropyrazolo[5,4-b]pyrimidino[5,4-e]pyridin-5-one-7-(p-

chloro)benzaldehyde hydrazone (13b). Pale light yellow crystals, from dioxane. Yield: 72\%. Mp. 310-311 ${ }^{\circ} \mathrm{C}$ (dec.); IR (KBr) cm ${ }^{-1}: 3320,3170(2 \mathrm{NH}), 1685(\mathrm{C}=\mathrm{O}), 1645(\mathrm{C}=\mathrm{N}),{ }^{1} \mathrm{H}-\mathrm{NMR}\left(\mathrm{DMSO}-\mathrm{d}_{6}\right)$ $\delta(\mathrm{ppm}): 1.25$ (t, $\left.J=7.4 \mathrm{~Hz}, 3 \mathrm{H}, \mathrm{NCH}_{2} \underline{\mathrm{CH}}_{3}\right), 2.88\left(\mathrm{~s}, 3 \mathrm{H}, \mathrm{CH}_{3}\right), 3.35$ (q, $\left.J=7.4 \mathrm{~Hz}, 2 \mathrm{H}, \mathrm{NCH}_{2} \mathrm{CH}_{3}\right)$, $5.98(\mathrm{~s}, 1 \mathrm{H}, \mathrm{CH}), 7.25-8.15(\mathrm{~m}, 14 \mathrm{H}, \mathrm{Ar}-\mathrm{H}), 9.15$ (s,1H, azomethine proton), 10.85 (brs, 1H, NH, $\mathrm{D}_{2} \mathrm{O}$ exchangeable), 12.75 (brs, $1 \mathrm{H}, \mathrm{NH}, \mathrm{D}_{2} \mathrm{O}$ exchangeable); Anal. Calcd. for $\mathrm{C}_{30} \mathrm{H}_{26} \mathrm{ClN}_{7} \mathrm{O}$ (536.03): $\mathrm{C}$, 67.22; H, 4.89; Cl, 6.61; N, 18.29; Found: C, 67.33; H, 4.94; Cl, 6.59; N, 18.41.

6-Ethyl-3-methyl-1,4-diphenyl-4,6,9-trihydropyrazolo[5,4-b]pyrimidino[5,4-e]pyridin-5-one-7-(pmethoxy)benzaldehyde hydrazone (13c). Pale white crystals from dioxane. Yield: 68\%. Mp. 216-218 ${ }^{\circ} \mathrm{C}$; IR (KBr)cm ${ }^{-1}:$ 3330, $3100(2 \mathrm{NH}), 1690(\mathrm{C}=\mathrm{O}), 1635(\mathrm{C}=\mathrm{N}),{ }^{1} \mathrm{H}-\mathrm{NMR}\left(\mathrm{DMSO}-\mathrm{d}_{6}\right) \delta(\mathrm{ppm}): 1.15(\mathrm{t}$, $\left.J=7.4 \mathrm{~Hz}, 3 \mathrm{H}, \mathrm{NCH}_{2} \underline{\mathrm{CH}}_{3}\right), 2.90\left(\mathrm{~s}, 3 \mathrm{H}, \mathrm{CH}_{3}\right), 3.30\left(\mathrm{q}, J=7.4 \mathrm{~Hz}, 2 \mathrm{H}, \mathrm{NCH}_{2} \mathrm{CH}_{3}\right), 3.94\left(\mathrm{~s}, 3 \mathrm{H}, \mathrm{OCH}_{3}\right)$ $5.88(\mathrm{~s}, 1 \mathrm{H}, \mathrm{CH}), 7.20-7.95(\mathrm{~m}, 14 \mathrm{H}, \mathrm{Ar}-\mathrm{H}), 9.50$ (s,1H, methylenic proton), 10.77 (brs, $1 \mathrm{H}, \mathrm{NH}, \mathrm{D}_{2} \mathrm{O}$ 
exchangeable), 11.45(brs, $1 \mathrm{H}, \mathrm{NH}, \mathrm{D}_{2} \mathrm{O}$ exchangeable). Anal. Calcd. for $\mathrm{C}_{31} \mathrm{H}_{2} \mathrm{~N}_{7} \mathrm{O}_{2}$ (531.61): C, 70.04; H, 5.50; N, 18.44; Found: C, 70.14; H, 5.59; N, 18.41 .

3.9. 6-Ethyl-3-methyl-7-(3-methyl-5-oxo(2-pyrazolinyl))-1,4-diphenyl-4,6,9-trihydropyrazolo[5,4-b]pyrimidino[5,4-e]pyridin-5-one (14)

A solution of compound $12(0.413 \mathrm{~g}, 0.001 \mathrm{~mol})$ and ethyl acetoacetate $(0.130 \mathrm{~g}, 0.001 \mathrm{~mol})$ in sodium ethoxide solution [prepared by dissolving sodium metal $(0.023 \mathrm{~g}, 0.001 \mathrm{~mol})$ in absolute ethanol $(30 \mathrm{~mL})$ ] was heated under reflux with stirring for $4 \mathrm{~h}$. The reaction mixture was allowed to cool and poured into cold water $(100 \mathrm{~mL})$ and neutralized by acetic acid, whereby a solid was precipitated, which was filtered off and crystallized from chloroform. Yield: $73 \%$. Mp. $231-233{ }^{\circ} \mathrm{C}$; IR $(\mathrm{KBr}) \mathrm{cm}^{-1}: 3315(\mathrm{NH}), 1698,1676(2 \mathrm{C}=\mathrm{O}), 1550(\mathrm{C}=\mathrm{N}),{ }^{1} \mathrm{H}-\mathrm{NMR}\left(\mathrm{CDCl}_{3}\right) \delta(\mathrm{ppm}): 1.25(\mathrm{t}$, $\left.J=7.4 \mathrm{~Hz}, 3 \mathrm{H}, \mathrm{NCH}_{2} \underline{\mathrm{CH}}_{3}\right), 2.33\left(\mathrm{~s}, 3 \mathrm{H}, \mathrm{CH}_{3}\right), 2.55\left(\mathrm{~s}, 2 \mathrm{H}, \mathrm{CH}_{2}\right), 2.88\left(\mathrm{~s}, 3 \mathrm{H}, \mathrm{CH}_{3}\right), 3.50$ (q, $J=7.4 \mathrm{~Hz}$, $\left.2 \mathrm{H}, \underline{\mathrm{NCH}}_{2} \mathrm{CH}_{3}\right), 5.88(\mathrm{~s}, 1 \mathrm{H}, \mathrm{CH}), 7.20-7.66$ (m, 10H, Ar-H), 10.85(brs, 1H, NH, D $2 \mathrm{O}$ exchangeable); ${ }^{13} \mathrm{C}-\mathrm{NMR}\left(\mathrm{CDCl}_{3}\right) \delta(\mathrm{ppm}): 11.33\left(\mathrm{CH}_{3}\right), 12.79\left(\mathrm{CH}_{3}\right), 24.37\left(\mathrm{CH}_{3}\right), 30.65\left(\mathrm{CH}_{2}\right), 34.78(\mathrm{CH} \mathrm{sp})$, $44.65\left(\mathrm{CH}_{2}\right.$ pyrazole), 103.65-162.95 (19C, sp ${ }^{2}$ carbon atoms), $163.66(\mathrm{C}=\mathrm{O}), 164.815(\mathrm{C}=\mathrm{O}$ pyrazole). Anal. Calcd. for: $\mathrm{C}_{27} \mathrm{H}_{25} \mathrm{~N}_{7} \mathrm{O}_{2}$ (479.53): C, 67.63; H, 5.25; N, 20.45; Found: C, 67.71; H, $5.29 ; \mathrm{N}, 20.41$.

\subsection{7-(3,5-Dimethylpyrazolyl)-6-ethyl-3-methyl-1,4-diphenyl-4,6,9-trihydropyrazolo[5,4-b]-} pyrimidine [5,4-e]pyridin-5-one (15)

A mixture of compound $12(0.413 \mathrm{~g}, 0.001 \mathrm{~mol})$ and acetylacetone $(0.1 \mathrm{~g}, 0.001 \mathrm{~mol})$ in absolute ethanol $(30 \mathrm{~mL})$ was stirred under reflux for $5 \mathrm{~h}$. The reaction mixture was allowed to cool to $0{ }^{\circ} \mathrm{C}$ for $3 \mathrm{~h}$. The precipitate was filtered off, dried and crystallized from ethanol as pale light crystals. Yield: 80\%. Mp. 222-224 ${ }^{\circ} \mathrm{C}$. IR $(\mathrm{KBr}) \mathrm{cm}^{-1}: 3225(\mathrm{NH}), 1700(\mathrm{C}=\mathrm{O}), 1650(\mathrm{C}=\mathrm{N}),{ }^{1} \mathrm{H}-\mathrm{NMR}\left(\mathrm{CDCl}_{3}\right)$ $\delta(\mathrm{ppm}): 1.27\left(\mathrm{t}, J=7.4 \mathrm{~Hz}, 3 \mathrm{H}, \mathrm{NCH}_{2} \underline{\mathrm{CH}}_{3}\right), 2.41$ (s, 3H, $\left.\mathrm{CH}_{3}\right), 2.43\left(\mathrm{~s}, 3 \mathrm{H}, \mathrm{CH}_{3}\right), 2.68\left(\mathrm{~s}, 3 \mathrm{H}, \mathrm{CH}_{3}\right)$, $3.63\left(\mathrm{q}, J=7.4 \mathrm{~Hz}, 2 \mathrm{H}, \mathrm{NCH}_{2} \mathrm{CH}_{3}\right), 5.77(\mathrm{~s}, 1 \mathrm{H}, \mathrm{CH}), 6.15(1 \mathrm{H}, \mathrm{CH}$ pyrazole $), 7.20-7.82(\mathrm{~m}, 10 \mathrm{H}$, $\mathrm{Ar}-\mathrm{H}), 10.65(\mathrm{~s}, 1 \mathrm{H}, \mathrm{NH}) ;{ }^{13} \mathrm{C}-\mathrm{NMR}\left(\mathrm{CDCl}_{3}\right) \delta(\mathrm{ppm}): 11.33\left(\mathrm{CH}_{3}\right), 12.79\left(\mathrm{CH}_{3}\right), 17.62\left(\mathrm{CH}_{3}\right), 24.37$ $\left(\mathrm{CH}_{3}\right), 30.65\left(\mathrm{CH}_{2}\right), 33.66\left(\mathrm{CH} \mathrm{sp}^{3}\right), 102.25-160.95$ (21C, sp ${ }^{2}$ carbon atoms), $162.90(\mathrm{C}=\mathrm{O})$. Anal. Calcd. for: $\mathrm{C}_{28} \mathrm{H}_{27} \mathrm{~N}_{7} \mathrm{O}$ (477.56): C, 70.42; H, 5.70; N, 20.53; Found: C, 70.52; H, 5.79; N, 20.61.

\subsection{7-(3-Amino-5-oxo(2-pyrazolinyl))-6-ethyl-3-methyl-1,4-diphenyl-4,6,9-tri-hydropyrazolo[5,4-b]-} pyrimidino[5,4-e]pyridin-5-one (16)

To a warmed ethanolic sodium ethoxide solution [prepared by dissolving sodium metal (0.023 g, $0.001 \mathrm{~mol})$ in absolute ethanol $(30 \mathrm{~mL})$ ] was added compound $12(0.413 \mathrm{~g}, 0.001 \mathrm{~mol})$ and ethyl cyanoacetate $(0.113 \mathrm{~g}, 0.001 \mathrm{~mol})$. The mixture was stirred under reflux for $12 \mathrm{~h}$, the reaction mixture was allowed to cool to room temperature, then poured into cold water $(100 \mathrm{~mL})$ and neutralized with acetic acid. The solid product was filtered off, washed with water, ethanol, dried and crystallized from ethanol as pale brown crystals. Yield: $85 \%$. Mp. $310-312{ }^{\circ} \mathrm{C}$. IR $(\mathrm{KBr}) \mathrm{cm}^{-1}: 3455,3350\left(\mathrm{NH}_{2}\right), 3220$ $(\mathrm{NH}), 1695(\mathrm{C}=\mathrm{O}), 1700(\mathrm{C}=\mathrm{O}), 1640(\mathrm{C}=\mathrm{N}),{ }^{1} \mathrm{H}-\mathrm{NMR}\left(\mathrm{CDCl}_{3}\right) \delta(\mathrm{ppm}): 1.31(\mathrm{t}, J=7.4 \mathrm{~Hz}, 3 \mathrm{H}$, $\left.\mathrm{NCH}_{2} \underline{\mathrm{CH}}_{3}\right), 2.71$ (s, $\left.3 \mathrm{H}, \mathrm{CH}_{3}\right), 3.45$ (s, $2 \mathrm{H}, \mathrm{CH}_{2}$ pyrazole), 3.65 (q, $\left.J=7.4 \mathrm{~Hz}, 2 \mathrm{H}, \mathrm{NCH}_{2} \mathrm{CH}_{3}\right), 6.01$ 
(s, 1H, CH), 7.15-7.89 (m, 10H, Ar-H), 10.25 (s, 1H, NH), 12.11 (brs, $\mathrm{NH}_{2}, \mathrm{D}_{2} \mathrm{O}$ exchangeable). Anal. Calcd. for: $\mathrm{C}_{26} \mathrm{H}_{24} \mathrm{~N}_{8} \mathrm{O}_{2}$ (480.52): C, 64.99; H, 5.03; N, 23.32; Found: C, 65.08; H, 5.10; N, 23.41 .

3.12. 11-Ethyl-8-methyl-6,9-diphenyl-4,5,9,11-tetrahydropyrazolo[5,4-b]1,2,4-triazolo[4',3'-2,1]pyrimidino[5,6-e]pyridin-10-one (17)

A mixture of compound $12(0.413 \mathrm{~g}, 0.001 \mathrm{~mol})$ and triethyl orthoformate $(0.192 \mathrm{~g}, 0.0013 \mathrm{~mol})$ in ethanol $(30 \mathrm{~mL})$ was refluxed in the presence of few drops of acetic acid for $3 \mathrm{~h}$. The solid product that separated from the hot mixture was filtered off, and recrystallized from acetic acid as yellow crystals. Yield: 56\%. Mp. $>300{ }^{\circ} \mathrm{C}$. IR $(\mathrm{KBr}) \mathrm{cm}^{-1}: 3315(\mathrm{NH}), 1695(\mathrm{C}=\mathrm{O}), 1650(\mathrm{C}=\mathrm{N}),{ }^{1} \mathrm{H}-\mathrm{NMR}\left(\mathrm{DMSO}-\mathrm{d}_{6}\right)$ $\delta(\mathrm{ppm}): 1.24\left(\mathrm{t}, J=7.4 \mathrm{~Hz}, 3 \mathrm{H}, \mathrm{NCH}_{2} \underline{\mathrm{CH}}_{3}\right), 2.71\left(\mathrm{~s}, 3 \mathrm{H}, \mathrm{CH}_{3}\right), 3.45$ (q, $\left.J=7.4 \mathrm{~Hz}, 2 \mathrm{H}, \mathrm{NCH}_{2} \mathrm{CH}_{3}\right)$, $6.15(\mathrm{~s}, 1 \mathrm{H}, \mathrm{CH}), 7.15-7.92(\mathrm{~m}, 10 \mathrm{H}, \mathrm{Ar}-\mathrm{H}), 8.55(\mathrm{~s}, 1 \mathrm{H}, \mathrm{CH}$ triazole), 11.05 (s, 1H, NH). Anal. Calcd. for $\mathrm{C}_{24} \mathrm{H}_{21} \mathrm{~N}_{7} \mathrm{O}$ (423.47): C, 68.07; H, 5.01; N, 23.15; Found: C, 68.16; H, 4.96; N, 23.22. MS $m / z(\%) 423.21\left(\mathrm{M}^{+}, 100\right)$.

\subsection{1-Ethyl-8-methyl-6,9-diphenyl-10-oxo-4,5,9,11-tetrahydropyrazolo[5,4-b] 1,2,4-triazolo[4',3'-} 2,1]pyrimidino[5,6-e]pyridin-3(2H)-thione (18)

A mixture of compound $12(2.06 \mathrm{~g}, 0.005 \mathrm{~mol})$ and carbon disulfide $(0.95 \mathrm{~g}, 0.005 \mathrm{~mol})$ in ethanolic sodium hydroxide $(10 \mathrm{~mL}, 10 \%)$ was heated on a water bath for $2 \mathrm{~h}$. The solvent was evaporated under reduced pressure, the residue was diluted with water $(30 \mathrm{~mL})$, acidified with $\mathrm{HCl}$. The solid product was filtered off and recrystallized from acetic acid as orange crystals. Yield: $69 \% \mathrm{Mp} .285-287{ }^{\circ} \mathrm{C}$. IR $(\mathrm{KBr}) \mathrm{cm}^{-1}: 3295(\mathrm{NH}), 1705(\mathrm{C}=\mathrm{O}), 1620(\mathrm{C}=\mathrm{N}),{ }^{1} \mathrm{H}-\mathrm{NMR}\left(\mathrm{DMSO}-d_{6}\right) . \delta(\mathrm{ppm}): 0.99(\mathrm{t}, J=7.4 \mathrm{~Hz}$, $\left.3 \mathrm{H}, \mathrm{NCH}_{2} \underline{\mathrm{CH}}_{3}\right), 2.45$ (s, 3H, $\left.\mathrm{CH}_{3}\right), 3.15$ (q, $\left.J=7.4 \mathrm{~Hz}, 2 \mathrm{H}, \mathrm{NCH}_{2} \mathrm{CH}_{3}\right), 5.65$ (s, 1H, CH), 7.30-7.99 (m, 10H, Ar-H), 10.75 (s, 1H, NH). Anal. Calcd. for: $\mathrm{C}_{24} \mathrm{H}_{21} \mathrm{~N}_{7} \mathrm{OS}$ (455.53): C, 63.28; H, 4.65; N, 21.52; S, 7.04; Found: C, 63.36; H, 4.72; N, 21.62.; S, 6.98. MS m/z (\%) $455.17\left(\mathrm{M}^{+}, 100\right)$.

\subsection{General Procedure for the Preparation of 19a-c}

These compounds were synthesized following a procedure analogous to that for compounds $7 \mathbf{a}-\mathbf{f}$ using a mixture of $18(0.44 \mathrm{~g}, 0.001 \mathrm{~mol})$, ethyl iodide and/or $\alpha$-haloketone $(0.001 \mathrm{~mol})$. The solid product separated from the hot mixture was filtered off, washed with water and recrystallized from ethanol.

3-Ethylthio-11-ethyl-8-methyl-6,9-diphenyl-4,5,9,11-tetrahydropyrazolo[5,4-b] 1,2,4-triazolo[4',3'-

2,1]pyrimidino[5,6-e]pyridin-10-one (19a). Yellow crystals. Yields: 63\%. Mp. 245-247 ${ }^{\circ} \mathrm{C}$. IR (KBr) $\mathrm{cm}^{-1}: 3305(\mathrm{NH}), 1705(\mathrm{C}=\mathrm{O}), 1630(\mathrm{C}=\mathrm{N}),{ }^{1} \mathrm{H}-\mathrm{NMR}\left(\mathrm{DMSO}_{6} \mathrm{~d}_{6}\right) \mathrm{ppm} \delta: 1.30(\mathrm{t}, J=7.4 \mathrm{~Hz}, 3 \mathrm{H}$, $\left.\mathrm{NCH}_{2} \underline{\mathrm{CH}}_{3}\right), 1.58\left(\mathrm{t}, J=7.4 \mathrm{~Hz}, 3 \mathrm{H}, \mathrm{SCH}_{2} \underline{\mathrm{CH}}_{3}\right), 2.69\left(\mathrm{~s}, 3 \mathrm{H}, \mathrm{CH}_{3}\right), 3.11$ (q, J=7.4 Hz, 2H, $\left.\underline{\mathrm{NCH}}_{2} \mathrm{CH}_{3}\right)$, 3.55 ( q, $\left.J=7.4 \mathrm{~Hz}, 2 \mathrm{H}, \underline{\mathrm{SCH}}_{2} \mathrm{CH}_{3}\right), 5.95$ (s, 1H, CH), 7.40-7.85 (m, 10H, Ar-H), 10.30 (s, 1H, NH, $\mathrm{D}_{2} \mathrm{O}$ exchangeable). ${ }^{13} \mathrm{C}-\mathrm{NMR}\left(\mathrm{DMSO}_{\mathrm{d}}\right) \delta(\mathrm{ppm}): 11.13\left(\mathrm{CH}_{3}\right), 12.50\left(\mathrm{CH}_{3}\right), 14.65\left(\mathrm{CH}_{3}\right), 29.75$ $\left(\mathrm{CH}_{2}\right), 35.60\left(\mathrm{CH} \mathrm{sp}^{3}\right), 42.80\left(\mathrm{CH}_{2}\right), 103.15-160.95$ (19C, sp ${ }^{2}$ carbon atoms), $162.20(\mathrm{C}=\mathrm{O})$. Anal. Calcd. for: $\mathrm{C}_{26} \mathrm{H}_{25} \mathrm{~N}_{7} \mathrm{OS}$ (483.59): C, 64.58; H, 5.21; N, 20.27; S, 6.63; Found: C, 64.69; H, 5.30; N, 20.32; S, 6.78. 
Ethyl-3-(11-ethyl-8-methyl-6,9-dipheny-10-oxo--4,5,9,11-tetrahydro-pyrazolo[5,4-b]1,2,4-triazolo[4',3'2,1]pyrimidino[5,6-e]pyridine)acetate (19b). Yellow crystals. Yield: 79\%. Mp. $189-191{ }^{\circ} \mathrm{C}$; IR (KBr) $\mathrm{cm}^{-1}: 3280(\mathrm{NH}), 1705(\mathrm{C}=\mathrm{O}), 1735(\mathrm{C}=\mathrm{O}), 1600(\mathrm{C}=\mathrm{N}),{ }^{1} \mathrm{H}-\mathrm{NMR}\left(\mathrm{DMSO}-\mathrm{d}_{6}\right) \delta(\mathrm{ppm}): 1.25(\mathrm{t}$, $\left.J=7.4 \mathrm{~Hz}, 3 \mathrm{H}, \mathrm{NCH}_{2} \underline{\mathrm{CH}}_{3}\right), 1.45\left(\mathrm{t}, J=7.4 \mathrm{~Hz}, 3 \mathrm{H}, \mathrm{SCH}_{2} \underline{\mathrm{CH}}_{3}\right), 2.70$ (s, 3H, $\left.\mathrm{CH}_{3}\right), 3.10$ (q, $J=7.4 \mathrm{~Hz}$, $2 \mathrm{H}, \underline{\mathrm{NCH}}_{2} \mathrm{CH}_{3}$ ), 3.80 (s, $\left.2 \mathrm{H}, \mathrm{SCH}_{2}\right), 4.15$ (q, $\left.J=7.4 \mathrm{~Hz}, 2 \mathrm{H}, \underline{\mathrm{SCH}}_{2} \mathrm{CH}_{3}\right), 6.15(\mathrm{~s}, 1 \mathrm{H}, \mathrm{CH}), 7.40-7.85$ (m, $10 \mathrm{H}, \mathrm{Ar}-\mathrm{H}), 11.03$ (s, $1 \mathrm{H}, \mathrm{NH}, \mathrm{D}_{2} \mathrm{O}$ exchangeable). Anal. Calcd. for: $\mathrm{C}_{28} \mathrm{H}_{27} \mathrm{~N}_{7} \mathrm{O}_{3} \mathrm{~S}$ (541.49): $\mathrm{C}$, 62.09; H, 5.02; N, 18.10; S, 5.92; Found: C, 62.23; H, 4.87; N, 18.19; S, 5.99.

11-Ethyl-3-(2-oxo-2-phenylethylthio)--8-methyl-6,9-dipheny-10-oxo-4,5,9,11-tetrahydro-pyrazolo[5,4b] 1,2,4-triazolo[4',3'-2,1]pyrimidino[5,6-e]pyridine-10-one (19c). Pale yellow crystals. Yield: 68\%. Mp. 272-274 ${ }^{\circ} \mathrm{C}$. IR (KBr) cm ${ }^{-1}: 3290(\mathrm{NH}), 1705(\mathrm{C}=\mathrm{O}), 1690(\mathrm{br}, \mathrm{C}=\mathrm{O}), 1600(\mathrm{C}=\mathrm{N}),{ }^{1} \mathrm{H}-\mathrm{NMR}$ $\left(\mathrm{DMSO}_{-} \mathrm{d}_{6}\right) \delta(\mathrm{ppm}): 1.18\left(\mathrm{t}, J=7.4 \mathrm{~Hz}, 3 \mathrm{H}, \mathrm{NCH}_{2} \underline{\mathrm{CH}}_{3}\right), 2.77\left(\mathrm{~s}, 3 \mathrm{H}, \mathrm{CH}_{3}\right), 3.32(\mathrm{q}, J=7.4 \mathrm{~Hz}, 2 \mathrm{H}$, $\left.\underline{\mathrm{NCH}}_{2} \mathrm{CH}_{3}\right), 4.35\left(\mathrm{~s}, 2 \mathrm{H}, \mathrm{SCH}_{2}\right), 5.95(\mathrm{~s}, 1 \mathrm{H}, \mathrm{CH}), 7.20-8.25(\mathrm{~m}, 15 \mathrm{H}, \mathrm{Ar}-\mathrm{H}), 10.80\left(\mathrm{~s}, 1 \mathrm{H}, \mathrm{NH}, \mathrm{D}_{2} \mathrm{O}\right.$ exchangeable). Anal. Calcd. for $\mathrm{C}_{32} \mathrm{H}_{27} \mathrm{~N}_{7} \mathrm{O}_{2} \mathrm{~S}$ (573.67): C, 67.01; H, 4.74; N, 17.09; S, 5.59; Found: C, 67.21; H, 4.83; N, 17.23.; S, 5.49. MS $m / z(\%) 573.57\left(\mathrm{M}^{+}, 100\right)$.

3.15. 4-Ethyl-7-methyl-6,9-diphenyl-4,6,10,11-tetrahydropyrazolo[5,4-b] 1,2,3,4-tetraazolo[1',5'-1,2]pyrimidino[5,6-e]pyridin-5-one (20)

A solution of sodium nitrite $(0.07 \mathrm{~g}, 0.001 \mathrm{~mol})$ in the least amount of water was added dropwise to an ice-cold solution of compound $12(0.413 \mathrm{~g}, 0.001 \mathrm{~mol})$ in acetic acid $(10 \mathrm{~mL})$ kept in an ice bath at $-5{ }^{\circ} \mathrm{C}$. The reaction mixture was allowed to stand overnight at room temperature, then it was poured into water $(100 \mathrm{~mL})$. The precipitate that formed was filtered off and crystallized from dioxane. It separated as pale yellow needles. Yield: $75 \%$. Mp. $>300{ }^{\circ} \mathrm{C}$; IR $(\mathrm{KBr}) \mathrm{cm}^{-1}: 3350(\mathrm{NH}), 2250\left(\mathrm{~N}_{3}\right)$, $1695(\mathrm{C}=\mathrm{O}), 1620(\mathrm{C}=\mathrm{N}) .{ }^{1} \mathrm{H}-\mathrm{NMR}\left(\mathrm{DMSO}_{\mathrm{d}}\right) \delta(\mathrm{ppm}): 1.33\left(\mathrm{t}, J=7.4 \mathrm{~Hz}, 3 \mathrm{H}, \mathrm{NCH}_{2} \underline{\mathrm{CH}}_{3}\right), 2.60$ (s, $\left.3 \mathrm{H}, \mathrm{CH}_{3}\right), 3.54$ (q, J=7.4 Hz, 2H, $\left.\mathrm{NCH}_{2} \mathrm{CH}_{3}\right), 5.85$ (s, 1H, CH), 7.20-7.90 (m, 10H, Ar-H), 11.25 (s, $1 \mathrm{H}, \mathrm{NH}, \mathrm{D}_{2} \mathrm{O}$ exchangeable). ${ }^{13} \mathrm{C}-\mathrm{NMR}\left(\mathrm{DMSO}_{-} \mathrm{d}_{6}\right) \delta(\mathrm{ppm}): 11.03\left(\mathrm{CH}_{3}\right), 12.35\left(\mathrm{CH}_{3}\right), 33.45(\mathrm{CH}$ $\left.\mathrm{sp}^{3}\right), 40.65\left(\mathrm{CH}_{2}\right), 101.75-160.15\left(18 \mathrm{C}, \mathrm{sp}^{2}\right.$ carbon atoms), $163.30(\mathrm{C}=\mathrm{O})$. Anal. Calcd. for $\mathrm{C}_{23} \mathrm{H}_{20} \mathrm{~N}_{8} \mathrm{O}$ (424.46): C, 65.08; H, 4.75; N, 26.40; Found: C, 65.19; H, 4.83; N, 26.55.

3.16. 5-Ethyl-8-methyl-1,2,7,10-tetraphenyl-5,7,11,12-tetrahydro-1H-pyrazolo[5,4-b] 1,2,4triazino[4',3'-2,1]-pyrimidino[5,6-e]pyridin-6-one (21)

A mixture of compound $12(0.413 \mathrm{~g}, 0.001 \mathrm{~mol})$ and benzoin $(0.21 \mathrm{~g}, 0.001 \mathrm{~mol})$ was heated under reflux in a mixture of pyridine and acetic anhydride $(20 \mathrm{~mL})(1: 1)$ for $5 \mathrm{~h}$. The reaction mixture was allowed to cool, poured onto ice cold water and neutralized with dilute $\mathrm{HCl}$, The solid product was filtered off and recrystallized from acetic as pale gray needles. Yield: $55 \%$. Mp. $>300{ }^{\circ} \mathrm{C}$; IR $(\mathrm{KBr})$ $\mathrm{cm}^{-1}: 3270(\mathrm{NH}), 1690(\mathrm{C}=\mathrm{O}), 1640(\mathrm{C}=\mathrm{N}) .{ }^{1} \mathrm{H}-\mathrm{NMR}\left(\mathrm{DMSO}_{-} \mathrm{d}_{6}\right) \delta(\mathrm{ppm}): 1.35(\mathrm{t}, J=7.4 \mathrm{~Hz}, 3 \mathrm{H}$, $\left.\mathrm{NCH}_{2} \underline{\mathrm{CH}}_{3}\right), 2.40\left(\mathrm{~s}, 3 \mathrm{H}, \mathrm{CH}_{3}\right), 3.15(\mathrm{~s}, 1 \mathrm{H}, \mathrm{CH}), 3.35$ (q, J = 7.4 Hz, 2H, $\left.\underline{\mathrm{NCH}}_{2} \mathrm{CH}_{3}\right), 6.05(\mathrm{~s}, 1 \mathrm{H}, \mathrm{CH})$, 7.20-7.90 (m, 20H, Ar-H), 10.40 (s, 1H, NH, D $2 \mathrm{O}$ exchangeable). ${ }^{13} \mathrm{C}-\mathrm{NMR}$ (DMSO-d $\left.\mathrm{d}_{6}\right) \delta(\mathrm{ppm})$ : $\left.11.03\left(\mathrm{CH}_{3}\right), 12.55\left(\mathrm{CH}_{3}\right), 32.35(\mathrm{CH} \mathrm{sp})^{3}\right), 38.70\left(\mathrm{CH}_{2}\right), 44.55\left(\mathrm{CH} \mathrm{sp}{ }^{3}\right), 105.80-161.85\left(31 \mathrm{C}, \mathrm{sp}^{2}\right.$ carbon atoms), $162.90(\mathrm{C}=\mathrm{O})$. Anal. Calcd. for $\mathrm{C}_{37} \mathrm{H}_{31} \mathrm{~N}_{7} \mathrm{O}$ (589.66): C, 75.35; H, 5.82; N, 16.19; Found: C, 75.73; H, 6.12; N, 16.53 . 


\subsection{General Procedure for the Preparation of 22a,b}

A mixture of compound $12(0.001 \mathrm{~mol})$ with chloroacetone or phenacyl bromide $(0.001 \mathrm{~mol})$ in DMF $(30 \mathrm{~mL})$ and drops of glacial acid $(0.2 \mathrm{~mL})$ was heated in $90{ }^{\circ} \mathrm{C}$ for $10 \mathrm{~h}$. The solid that precipitated upon cooling was filtered off and crystallized from ethanol.

5-Ethyl-1,8-dimethyl-7,10-diphenyl-5,7,11,12-tetrahydro-3H-pyrazolo[5,4-b] 1,2,4-triazino[4',3'-2,1]pyrimidino[5,6-e]pyridin-6-one (22a). Pale white crystals. Yield: $51 \%$. Mp. $241-243{ }^{\circ} \mathrm{C}$ (dec.). IR $(\mathrm{KBr}) \mathrm{cm}^{-1}: 3310,3290(2 \mathrm{NH}), 1685(\mathrm{C}=\mathrm{O}) 1630(\mathrm{C}=\mathrm{N}),{ }^{1} \mathrm{H}-\mathrm{NMR}\left(\mathrm{DMSO}-\mathrm{d}_{6}\right) \delta(\mathrm{ppm}): 1.20(\mathrm{t}$, $\left.J=7.4 \mathrm{~Hz}, 3 \mathrm{H}, \mathrm{NCH}_{2} \underline{\mathrm{CH}}_{3}\right), 2.33\left(\mathrm{~s}, 3 \mathrm{H}, \mathrm{CH}_{3}\right), 2.65\left(\mathrm{~s}, 3 \mathrm{H}, \mathrm{CH}_{3}\right), 3.54$ (q, J=7.4 Hz, 2H, $\left.\underline{\mathrm{NCH}}_{2} \mathrm{CH}_{3}\right)$, $5.70(\mathrm{~s}, 1 \mathrm{H}, \mathrm{CH}), 7.15-7.95(\mathrm{~m}, 10 \mathrm{H}, \mathrm{Ar}-\mathrm{H}), 9.25(\mathrm{~s}, 1 \mathrm{H}$, triazine $), 10.80\left(\mathrm{~s}, 1 \mathrm{H}, \mathrm{NH}, \mathrm{D}_{2} \mathrm{O}\right.$ exchangeable), 10.95 (s, 1H, NH, D $2 \mathrm{O}$ exchangeable). ${ }^{13} \mathrm{C}-\mathrm{NMR}$ (DMSO-d $\mathrm{d}_{6} \delta(\mathrm{ppm}): 11.90\left(\mathrm{CH}_{3}\right)$, $12.45\left(\mathrm{CH}_{3}\right), 16.55\left(\mathrm{CH}^{3}\right), 30.98\left(\mathrm{CH}_{2}\right), 34.95\left(\mathrm{CH} \mathrm{sp}{ }^{3}\right), 36.15\left(\mathrm{CH} \mathrm{sp}{ }^{3}\right), 104.90-161.85\left(19 \mathrm{C}, \mathrm{sp}^{2}\right.$ carbon atoms), $162.90(\mathrm{C}=\mathrm{O})$. Anal. Calcd. for $\mathrm{C}_{26} \mathrm{H}_{25} \mathrm{~N}_{7} \mathrm{O}$ (451.52): C, 69.16; H, 5.58; N, 21.71; Found: C, 69.24; H, 5.73; N, 21.81.

5-Ethyl-8-methyl-1,7,10-triphenyl-5, 7,11,12-tetrahydro-3H-pyrazolo[5,4-b] 1,2,4-triazino[4',3'-2,1]pyrimidino[5,6-e]pyridin-6-one (22b). Pale white crystals. Yield: $63 \%$. Mp. $>300{ }^{\circ} \mathrm{C}, \mathrm{IR}(\mathrm{KBr}) \mathrm{cm}^{-1}$ : 3400, $3390(2 \mathrm{NH}), 1685(\mathrm{C}=\mathrm{O}) 1630(\mathrm{C}=\mathrm{N}),{ }^{1} \mathrm{H}-\mathrm{NMR}\left(\mathrm{DMSO}_{-} \mathrm{d}_{6}\right) \delta(\mathrm{ppm}): 1.19(\mathrm{t}, J=7.4 \mathrm{~Hz}, 3 \mathrm{H}$, $\left.\mathrm{NCH}_{2} \underline{\mathrm{CH}}_{3}\right), 2.55$ (s, 3H, $\left.\mathrm{CH}_{3}\right), 4.04$ (q, $\left.J=7.4 \mathrm{~Hz}, 2 \mathrm{H}, \mathrm{NCH}_{2} \mathrm{CH}_{3}\right), 6.10$ (s, $\left.1 \mathrm{H}, \mathrm{CH}\right), 7.15-8.15$ (m, 15H, Ar-H), $9.30(\mathrm{~s}, 1 \mathrm{H}$, triazine), 10.75 (s, 1H, NH, D $2 \mathrm{O}$ exchangeable), 11.25 (s, 1H, NH, D $2 \mathrm{O}$ exchangeable). Anal. Calcd. for: $\mathrm{C}_{31} \mathrm{H}_{27} \mathrm{~N}_{7} \mathrm{O}$ (513.59): C, 72.50; H, 5.30; N, 19.09; Found: C, 72.64; H, 5.41; N, 19.16. MS $m / z(\%) 513.48\left(\mathrm{M}^{+}, 100\right)$.

\subsection{Antimicrobial Activity}

The antimicrobial activity of 10 new chemical compounds was tested in vitro against six bacterial species obtained from contaminated soil, water and food substances (Staphylococcus aureus [AUMC No. B-54], Bacillus cereus [AUMC No. B-52], Micrococcus luteus (+ve) [AUMC NoB-112], Escherichia coli [AUMC No. B-53], Pseudomonas aeruginosa [AUMC No. B-73] and Serratia marcescens [AUMC No. B-55]. They were also tested against six fungal species which are involved in human and animal diseases (Trichophyton rubrum [AUMC No. 1804], Candida albicans [AUMC No. 418], Geotrichum candidum [AUMC No. 226], Scopulariopsis brevicaulis[AUMC No. 729] and Aspergillus flavus[AUMC No. 3214] or plant diseases (Fusarium oxysporum [AUMC No. 5119]. These strains are common contaminants of the environment in Egypt and some of all microbial strains were kindly provided by the Assiut University Mycological Centre (AUMC). To prepare inocula for bioassay, bacterial strains were individually cultured for $48 \mathrm{~h}$ in $100 \mathrm{~mL}$ conical flasks containing $30 \mathrm{~mL}$ nutrient broth medium. Fungi were grown for 7 days in $100 \mathrm{~mL}$ conicals containing $30 \mathrm{~mL}$ Sabouraud's dextrose broth. Bioassay was done in $10 \mathrm{~cm}$ sterile plastic Petri plates in which microbial suspension $(1 \mathrm{~mL} /$ plate) and $15 \mathrm{~mL}$ appropriate agar medium $(15 \mathrm{~mL} /$ plate $)$ were poured. Nutrient agar and Sabouraud's dextrose agar were respectively used for bacteria and fungi. After solidification of the media, $5 \mathrm{~mm}$ diameter cavities were cut in the solidified agar (4 cavities/plate) using sterile cork borer. Chemical compounds dissolved in DMSO at $2 \% \mathrm{w} / \mathrm{v}(=20 \mathrm{mg} / \mathrm{mL})$ were pipetted in the cavities 
(20 $\mu \mathrm{L} /$ cavity). Cultures were then incubated at $28{ }^{\circ} \mathrm{C}$ for $48 \mathrm{~h}$ in case of bacteria and up to 7 days in case of fungi. Results were read as the diameter (in $\mathrm{mm}$ ) of inhibition zone around cavities [29]. To determine the minimum inhibitory concentrations (MICs), chemical compounds giving positive results were diluted with DMSO to prepare a series of descending concentrations down to $0.02 \mathrm{mg} / \mathrm{mL}$. Diluted chemicals were similarly assayed as mentioned before and the least concentration (below which no activity) was recorded as the MIC.

\section{Conclusions}

In conclusion, we have reported the synthesis of some novel heterocyclic pyrazolopyrimidinopyridines and related compounds. Ten of the newly synthesized compounds have been screened for their biological activities against three Gram positive, three Gram negative bacteria, as well as six fungal strains. Most of the tested compounds showed activities against the strains used. Compound 19c proved to be the most potent compound of all those used.

\section{Supplementary Materials}

Supplementary materials can be accessed at: http://www.mdpi.com/1420-3049/17/12/14464/s1.

\section{Acknowledgements}

The authors would like to express their gratitude to R. M. Mahfouz, Professor of Inorganic Chemistry at Assiut Univerisity for running the ${ }^{13} \mathrm{C}-\mathrm{NMR}$ analysis at King Saoud University, Saudi Arabia.

\section{References}

1. Ganem, B. Strategies for innovation in multicomponent reaction design. Acc. Chem. 2009, 42, 463-472.

2. Padwa, A. Domino reactions of rhodium (II) carbenoids for alkaloid synthesis. Chem. Soc. Rev. 2009, 38, 3072-3081.

3. Ali, R.; Amir, T.M.; Morteza, R.; Aram, R. Novel three-component reaction of a secondary amine and a 2-hydroxybenzaldehyde derivative with an isocyanide in the presence of silica gel: an efficient one-pot synthesis of benzo[b]furan derivatives. Tetrahedron Lett. 2009, 50, 5625-5627.

4. Zeinab, Z.; Mehdi, K.; Ali, R.; Alireza, F.; Ali, S.; Katarzyna, Ś; Tadeusz Lis, A.S. Synthesis of functionalized furo[3,2-c] coumarins via a one-pot oxidative pseudo three-component reaction in poly(ethylene glycol. Tetrahedron 2012, 68, 6721-6726.

5. Domling, A. Multicomponent reactions. Chem. Rev. 2006, 106, 17-89.

6. D'Souza, D.M.; Muller, T.J. Multi-component syntheses of heterocycles by transition-metal catalysis. J. Chem. Soc. Rev. 2007, 36, 1095-1108.

7. Ali, R.; Ali, S. Iminophosphorane-mediated one-pot synthesis of 1,3,4-oxadiazole Derivatives. ARKIVOC 2008, xvi, 235-242. 
8. Ali, S.; Ali, R.; Nouri, B.; Richard, W. The reaction of ( $N$-isocyanimino)triphenylphosphorane with dialkyl acetylenedicarboxylates in the presence of 1,3-diphenyl-1,3-propanedione: A novel three-component reaction for the stereoselective synthesis of dialkyl (Z)-2-(5,7-diphenyl-1,3,4oxadiazepin-2-yl)-2-butenedioates. Tetrahedron Lett. 2007, 48, 2617-2620.

9. Ali, S.; Ali, R. The reaction of ( $N$-isocyanimino)triphenylphosphorane with benzoic acid derivatives: A novel synthesis of 2-aryl-1,3,4-oxadiazole derivatives. Tetrahedron Lett. 2007, 48, 1549-1551.

10. Shore, G.; Yoo, W.J.; Li, C.J.; Organ, M. Propargyl amine synthesis catalysed by gold and copper thin films using microwave assistant, continuous flow organic synthesis. Chem. Eur. J. 2010, 16, $126-133$.

11. Hardy, C.R. The chemistry of pyrazolopyridine. Adv. Heterocyl. Chem. 1984, 36, 343-409.

12. Elnagdi, M.H.; Elgemeie, G.H.; El-Moghayar, R.M.H. Chemistry of pyrazolopyrimidines. Adv. Heterocycl. Chem. 1987, 41, 319-376.

13. Jiaro, Q.; Jaime, P.; Silva, C.; Rodrigo, A.; Braulio, I.; Manuel, N.; Justo, C.; Mike, H. Solvent free synthesis of fused pyrazolo [1,5-a]pyrimidines by reaction of 5-amino-1- $H$-pyrazoles and ß-triketones. Open Org. Chem. J. 2008, 2, 92-99.

14. Jiaro, Q.; Jaime, P.;Rodrigo, A.; Manuel, N.; Justo, C.; Mike, H. Regioselective synthesis of novel substituted pyrazolo[1,5-a]pyrimidines under solvent-free conditions. Tetrahedron Lett. 2008, 49, 6254-6256.

15. Jiaro, Q.; Jaime, P.; Hugo, S.; Rodrigo, A.; Braulio, I.; Manuel, N.; Justo, C. Regioselective synthesis of fused benzopyrazolo[3,4-b]quinolines under solvent-free conditions. Tetrahedron Lett. 2007, 48, 1987-1990.

16. Aly, M.F.; El-Naggar, G.M.; El-Emary, T.I.; Girgg, R.; Metwally, S.A.; Sivagnanam, S. X=Y-ZH Compounds as potential 1,3-Dipoles part 41. Azomethine Ylide Formation from the reaction of -Amino acids and esters wth Alloxan (Strecker Degradation) and with 1-phenyl-3-methyl pyrazoline 4,5-dione. Tetrahedron 1994, 50, 895-906.

17. Selleri, S.; Burni, F.; Castanzo, A.; Gueririni, G.; Malmbeg-Aiello, P.; lavarone, G.; Martini, C. Synthesis and preliminary evaluation of pyrazolo[1,5-a]pyrido[3,4-e]pyrimidin-6-(7H)-ones and related comounds as benzodiazepine receptor ligands and anticonvulsant agents. Eur. J. Med. Chem. 1992, 27, 985-990.

18. Poreba, K.; Wietrzyk, J.; Opolski, A. Synthesis and antiproliferative activity in vitro of new 2,3 or 4 substituted pyrido[2',3':3,4]pyrazolo[1,5-a]pyrimidines. Acta Pol. Pharm. 2006, 63, 189-194.

19. Ismail, M.M.F.; Ammar, Y.A.; El-Zahaby, H.S.A.; Eisa, S.I.; Barakat, S.E. Synthesis of Novel-1pyrazolylpyridinopyrimidin2-ones as potential Anti-inflammatory and Analgesic Agent. Arch. Pharm. Chem. Life Sci. 2007, 340, 476-481.

20. Bi, Y.; Stoy, P.; He, B.; Adam, L.; Krupinski, J.; Normandin, D.; Pongrac, R.; Seliger, L.; Waston, A.; Macor, J.E. The discovery of novel, potent and selective PDE inhibitors. Bioorg. Med. Chem. Lett. 2001, 11, 2461-2464.

21. Ahluwalia, V.K.; Dahiya, A.; Garg, V. Reaction of 5-amino-4-formyl-3-methyl(or phenyl)-1phenyl-1H-pyrazoles with active methylene compounds: Synthesis of fused heterocyclic rings. Indian J. Chem. 1997, 36B, 88-90 and references sited therein. 
22. Metwally, S.A.; El-Naggar, G.M.; El-Emary, T.I. Reactions of 4-(Dicyanomethylene)-3-methyl1-phenyl-2-pyrazolin-5-one towards methylene comoponds. Liebgs Ann. Chem. 1991, 62, 961-962.

23. Metwally, S.A.; El-Naggar, G.M.; Younis, M.I.; Elnagdi, M.H.; El-Emary, T.I. Reactions of 4-(Dicyanomethylene)-3-methyl-1-phenyl-2-pyrazolin-5- one towards Amines and Phenols. Liebgs Ann. Chem. 1989, 40, 1037-1040.

24. El-Emary, T.I.; El-Dean, A.M.; El-Kashef, H.S. Facile synthesis of some new pyrazolo [3,4- $b$ ] pyrazines and their antifungal activity. II Farmaco 1998, 53, 383-388.

25. El-Kashef, H.S.; El-Emary, T.I.; Gasquet, M.; Timon-David, M.J.; Vanele, P. New pyrazolo [3,4-b] pyrazines: Synthesis and biological activity. Pharmazie 2000, 55, 572-577.

26. El-Emary, T.I.; El-kashef, H.S.; Hussein, A.M. New Polycyclic Azines Derived from Pyrazolo [3,4-b] pyridine. Pharmazie 2000, 55, 356-358.

27. Hussein, A.M.; El-Emary, T.I. Polycyclic Pyrazoles: Routes to New Pyrazoloazines. J. Chem. Res. 1998, 228-236.

28. El-Emary, T.I.; Bakhite, E.A. Synthesis and biological screening of new 1,3- diphenylpyrazoles with different moieties at position-4. Pharmazie 1999, 2, 106-111.

29. El-Emary, T.I.; Abdel-Mohsen, Sh.A. Synthesis and antimicrobial activity of some new 1,3diphenyl pyrazoles bearing pyrimidine, pyrimidinethione, thiazolopyrimidine, triazolopyrimidine, thio and alkyl-thiotriazolpyrimidinone moieties at 4- position. Phosphorous Sulfur Silicon 2006, 181, 2459-2474.

30. El-Emary, T.I.; Khalil, A.; Ali, G.A.; El-Adasy, A.A. A facile synthesis of some new Thiazolo[3,2-a]pyridines containing pyrazolyl moiety and their antimicrobial activity. Phosphorous Sulfur Silicon 2005, 180, 19-30.

31. El-Emary, T.I. Synthesis of newly substituted pyrazoles and substituted pyrazolo[3,4-b]pyridines based on 5-amino-3-methyl-1-phenylpyrazole. J. Chin. Chem. Soc. 2007, 54, 507-518.

32. El-Emary, T.I. Synthesis, reactions and biological activity of some new pyrazolo [3,4-b] pyrazines. J. Chin. Chem. Soc. 2006, 53, 391-401.

33. El-Emary, T.I. Synthesis of some newly condensed and uncondensed pyrazolo[3,4-b]pyridines. Assiut Univ. J. Chem. 2006, 35, 45-63.

34. Jairo, Q.; Diana, M.; Braulio, I.; Ridrigo, A.; Manuel, N.; Adolfo, S.; Justo, C.; John, N. Regioselective synthesis of 4,7,8,9-tetrahydro- $2 H$-pyrazolo[3,4- $b$ ]quinolin-5(6H)-ones. Mechanism and structural analysis. Tetrahedron 2001, 57, 6947-6953.

35. Bazgir, A; Khanaposhanti, M.; Sooki, A. One-pot synthesis and antibacterial activities of pyrazolo[4',3':5,6]pyrido[2,3-d]pyrimidine-dione derivatives. Bioorg. Med. Chem. Lett. 2008, 18, 5800-5803.

36. Chebanov, V.A.; Sakhno, Y.I.; Desenk, S.M.; Chernenko, V.N.; Musatov, V.I.; Shishkina, S.V.; Shishkin, O.V.; Kappe, O. Cyclocondensation reactions of 5-aminopyrazoles, pyruvic acids and aldehydes. Multicomponent approaches to pyrazolopyridines and related products. Tetrahedron 2007, 1229-1242.

37. Shaabani, A.; Seyyedhamez, M.; Maleki, A.; Behnan, M.; Rezazdeh, F. Synthesis of fully substituted pyrazolo[3,4-b]pyridine-5-carboxamide derivatives via a one-pot four-component reaction. Tetrahedron Lett. 2009, 50, 2911-2913. 
38. Balamurugan, K.; Perumal, S.; Menedez, J.C. New four-component reaction in water: a convergent approach to the metal-free synthesis of spiro[indoline/ acenaphthylene-3,4'pyrazolo[3,4-b]pyridine derivatives. Tetrahedron 2011, 67, 3201-3208.

39. Shawali, A.S.; Elghandour, A.H.; Sayed, A.R.A novel one-pot synthesis of 3-arylazo[1,2,4]triazolo[4,3-a]pyrimidin-5-(1H)-ones. Synth. Commun. 2001, 31, 731-740.

40. Bedford, G.R.; Taylor, P.J.; Webb, G.A. ${ }^{15} \mathrm{~N}-\mathrm{NMR}$ studies of guanidines. II-The fused-in guanidine unit of some oxoheterocycles: A combined ${ }^{15} \mathrm{~N}-\mathrm{NMR},{ }^{13} \mathrm{C}-\mathrm{NMR}$ and IR study. Magn. Res. Chem. 1995, 33, 389-394.

41. Elguero, J.; Goya, P.; Martinez, A.; Rozas, I. On the Tautomerism of 2-Phenacyl-4-pyrimidinones and Related Compounds. Chem. Ber. 1989, 122, 919-924.

42. Greenhill, J.V.; Ismail, M.J.; Bedford, G.R.; Edwards, P.N.; Taylor, P.J. Conformational and tautmeric studies of acyl guanidines Part 2. Vibrations and C-13 nuclear magnetic resonance spectroscopy. J. Chem. Soc. Perkin Trans. 1985, 2, 1265-1274.

43. Reiter, J.; Bongo, L.; Dyortsok, P. On triazoles XI. structure elucidation of isomeric 1,2,4-triazolopyrimidinones. Tetrahedron 1987, 43, 2497-2504.

44. Rami, V.J. One-Pot Synthesis of Mono- and Dinitro-1,2,4-triazino[3,2-b]benzothiazoles. Liebigs Ann. Chem. 1988, 11, 1089-1090.

45. Heinisch, G.; Holzer, W. Pyrazoles 3. N-1 Protected 4-Substituted Pyrazoles-Synthesis and Nmr Investigation. Heterocycles 1988, 27, 2443-2457.

46. Khalil, Z.H.; Geies, A.A. Synthesis and reactions of some thieno[2,3- $d]$ pyrimidine derivatives. Phosphorus Sulfur Silicon 1991, 60, 223-231.

47. Kwon-Chung, K.J.; Bennett, J.W. Principles of antifungal and antibacterial therapy. Med. Mycol. Lea Febiger. Philadel. 1992, 81-102.

Sample Availability: Samples of the compounds are available from the authors.

(C) 2012 by the authors; licensee MDPI, Basel, Switzerland. This article is an open access article distributed under the terms and conditions of the Creative Commons Attribution license (http://creativecommons.org/licenses/by/3.0/). 